\title{
Systematic extraction of crystal electric-field effects and quantum magnetic model parameters in triangular rare-earth magnets
}

\author{
Christopher A. Pocs, ${ }^{1}$ Peter E. Siegfried, ${ }^{1,{ }^{*}}$ Jie Xing, ${ }^{2}$ Athena S. Sefat,${ }^{2}$ Michael Hermele,,${ }^{1,3}$ B. Normand, ${ }^{4,5}$ \\ and Minhyea Lee (1) ${ }^{1}$ \\ ${ }^{1}$ Department of Physics, University of Colorado, Boulder, Colorado 80309, USA \\ ${ }^{2}$ Materials Science and Technology Division, Oak Ridge National Laboratory, Oak Ridge, Tennessee 37831, USA \\ ${ }^{3}$ Center for Theory of Quantum Matter, University of Colorado, Boulder, Colorado 80309, USA \\ ${ }^{4}$ Paul Scherrer Institute, CH-5232 Villigen-PSI, Switzerland \\ ${ }^{5}$ Institute of Physics, Ecole Polytechnique Fédérale de Lausanne (EPFL), CH-1015 Lausanne, Switzerland
}

(Received 7 October 2021; accepted 29 November 2021; published 21 December 2021)

\begin{abstract}
A primary goal at the interface of theoretical and experimental quantum magnetism is the investigation of exotic spin states, mostly notably quantum spin liquids (QSLs) that realize phenomena including quasiparticle fractionalization, long-ranged entanglement, and topological order. Magnetic rare-earth ions go beyond the straightforward paradigm of geometrical frustration in Heisenberg antiferromagnets by introducing competing energy scales, and in particular their strong spin-orbit coupling creates multiple split crystal electric-field (CEF) levels, leading to anisotropic effective spin models with intrinsic frustration. While rare-earth delafossites have a triangular-lattice geometry and thus have gained recent attention as candidates for hosting spin-1/2 QSL physics, the reliable extraction of effective spin models from the initial many-parameter CEF spectrum is a hard problem. Using the example of $\mathrm{CsYbSe}_{2}$, we demonstrate the unambiguous extraction of the Stevens operators dictating the full CEF spectrum of $\mathrm{Yb}^{3+}$ by translating these into parameters with a direct physical interpretation. Specifically, we combine low-field susceptibility measurements with resonant torsion magnetometry (RTM) experiments in fields up to $60 \mathrm{~T}$ to determine a sufficiently large number of physical parameters-effective Zeeman splittings, anisotropic van Vleck coefficients, and magnetotropic coefficients- that the set of Stevens operator coefficients is unique. Our crucial identification of the strong corrections to the Zeeman splitting of Kramers doublets as van Vleck coefficients has direct consequences for the interpretation of all anisotropic magnetic susceptibility measurements. Our results allow us to determine the nature and validity of an effective spin-1/2 model for $\mathrm{CsYbSe}_{2}$, to provide input for theoretical studies of such models on the triangular lattice, and to provide additional materials insight into routes for achieving magnetic frustration and candidate QSL systems in rare-earth compounds.
\end{abstract}

DOI: 10.1103/PhysRevResearch.3.043202

\section{INTRODUCTION}

The quantum spin liquid (QSL) is a nonmagnetic manybody ground state in which the spin correlations have long-ranged quantum entanglement [1]. Despite many theoretical studies of these enigmatic phases, which have served to drive detailed investigations of a wide range of magnetic compounds, neither a universally agreed QSL phase in a real material nor an unambiguous set of experimental QSL criteria has yet emerged. The strong quantum fluctuations responsible for producing exotic spin states are a consequence of generalized magnetic frustration, which leads to a highly de-

\footnotetext{
*Present address: Department of Physics, George Mason University, Fairfax VA 22030.

Published by the American Physical Society under the terms of the Creative Commons Attribution 4.0 International license. Further distribution of this work must maintain attribution to the author(s) and the published article's title, journal citation, and DOI.
}

generate manifold of competing states. Early examples of material realizations of candidate models for hosting QSL states were based on geometrical frustration in structures with triangular motifs, including kagome [2], pyrochlore [3,4], and triangular lattices [5-8], mostly of real $S=1 / 2$ spins.

Magnetic insulators with strong spin-orbit coupling are now widely recognized as a platform for extending very significantly the nature of frustration and the variety of quantum many-body phases (including QSLs) and phenomena that can be realized. When compared to spin-1/2 magnetic insulators based on $3 d$ ions, these systems tend to exhibit complex microscopic physics even at the single-ion level. Magnetic materials containing $5 d$ and $4 d$ transition-metal ions possess interactions that are anisotropic in both spin space and real space, leading to complex phenomenology in pyrochlore systems $[3,9,10]$ and in proximate Kitaev materials [11-15]. Compounds based on $4 f$ rare-earth ions that combine the geometric frustration of pyrochlore and triangular lattices with strong spin-orbit coupling have also provided fertile ground for quantum magnetism research [16-19]. However, in these materials a detailed understanding of the microscopic physics 
is a prerequisite for developing effective spin models that serve as a basis for theories of many-body phenomena.

In insulating $4 f$ materials, the key to a microscopic description of the magnetic interactions is a reliable determination of the single-ion crystal electric-field (CEF) Hamiltonian $\left(\mathcal{H}_{\mathrm{CEF}}\right)$ [3,4]. In many rare-earth compounds, CEF effects split the degeneracy of the ground multiplet of the total angular momentum, $J$. For half-odd-integer $J$, the result is a set of Kramers doublets. At sufficiently low temperatures, a restriction of the dynamics to the lowest such doublet may be invoked to justify using an effective pseudospin-1/2 model [16,20-22]. From an experimental standpoint, this allows a significant diversification of both materials and magnetic phenomena, while on the theoretical side a minimal model may be relatively simple, or even a realization of one of the paradigm models in frustrated magnetism.

The leading system-specific parameters in $\mathcal{H}_{\mathrm{CEF}}$ are commonly determined by inelastic neutron scattering (INS), which provides direct information about the spectrum of CEF multiplets. However, there are in general more symmetryallowed parameters in $\mathcal{H}_{\mathrm{CEF}}$ than there are gaps between CEF multiplets, resulting in an underdetermined fit of the CEF parameters and hence to uncertainties in the appropriate pseudospin- $1 / 2$ model that can be qualitative rather than merely quantitative corrections. Methods allowing the unambiguous determination of $\mathcal{H}_{\mathrm{CEF}}$ with higher reliability are thus very desirable. In this paper, we present a high-fidelity determination of the CEF parameters of a selected $4 f$ system, obtained by using a combination of low-field magnetic susceptibility and high-field (up to $60 \mathrm{~T}$ ) resonant torsion magnetometry (RTM) measurements.

Yb-based triangular-lattice compounds offer an excellent combination of geometric frustration, quasi-two-dimensional nature, half-odd-integer $J$, and a strongly split CEF spectrum that suggests the validity of pseudospin- $1 / 2$ models of quantum magnetism. In particular, the family of $A \mathrm{YbX} X_{2}$ delafossites (with $A=\mathrm{Na}$, Cs and $X=\mathrm{O}, \mathrm{S}, \mathrm{Se}$ ) has attracted intensive interest [21-29], and a very recent summary was compiled in Ref. [30]. Unlike the material $\mathrm{YbMgGaO}_{4}$ [31-36], they are free from potential site disorder $[37,38]$ and no members of the family have been found to exhibit magnetic ordering at temperatures down to tens of $\mathrm{mK}$ [30], while several have been reported to exhibit continua of low-energy magnetic excitations $[21,22,39]$. Their magnetic response is highly anisotropic between the in- and out-of-plane directions, providing a valuable opportunity to use the magnetic field to vary the free-energy landscape. Thus the trianguar-lattice delafossites present an ideal test case for unambiguous fitting of the field-induced CEF spectra and subsequent establishment of the effective spin- $1 / 2$ states in rare-earth compounds.

As an example material we focus on $\mathrm{CsYbSe}_{2}$ [Figs. 1(a) and 1(b)]. The complete CEF spectrum is specified by the coefficients of six Stevens operators, and thus the challenge is to measure enough independent physical quantities beyond the low-energy limit. From the susceptibility, we extract not only the linear Zeeman coefficients for both of the primary, high-symmetry field directions but also the van Vleck (VV) coefficients for the ground doublet, which are the second-order corrections (i.e., quadratic in the applied field). While these VV coefficients are often used to describe the
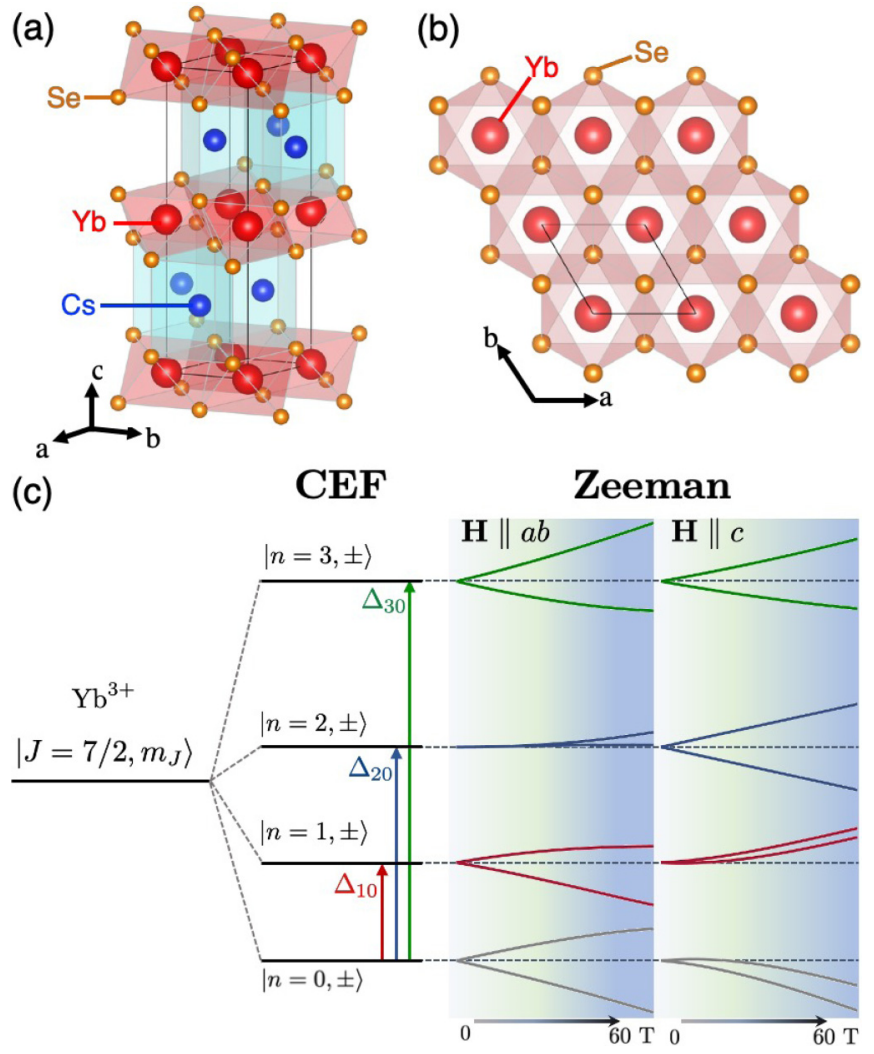

FIG. 1. Crystal structure and CEF spectrum. (a) $\mathrm{CsYbSe}_{2}$ adopts the $P 6_{3} / m m c$ space group. (b) $\mathrm{Yb}^{3+}$ (red) triangular layers formed by edge-sharing $\mathrm{YbSe}_{6}$ octahedra are separated by layers of $\mathrm{Cs}^{+}$ ions. (c) Schematic origin of the CEF energy spectrum of $\mathrm{Yb}^{3+}$. The CEF interactions allowed by the $D_{3 d}$ site symmetry split the $\mathrm{Yb}^{3+}$ ground-state manifold into four Kramers doublets. The application of a magnetic field lifts the doublet degeneracy in a spatially anisotropic manner whose leading nonlinear contributions are captured by the van Vleck coefficients defined in Eq. (7).

magnetic response of rare-earth compounds in terms of an additive contribution to the susceptibility $[25,40]$, in fact they are embedded in a nontrivial way in the full expression for this quantity. We will show how the multiple roles of the groundstate VV coefficients and their significance as stand-alone physical quantities, with direct implications for the high-field magnetic anisotropy, have yet to be appreciated in connection with CEF fitting. They facilitate the bridge to the field range covered by our RTM measurements, from which we extract the full field and temperature dependence of the magnetotropic coefficients for the same two high-symmetry field directions. These round out a complete set of eight observables, allowing us to determine a unique set of microscopic CEF parameters and thus the full CEF spectrum. As Fig. 1(c) makes clear, our results reveal an intricate energy landscape and level-repulsion behavior in the CEF spectrum of $\mathrm{CsYbSe}_{2}$ up to high magnetic field values and for both field directions.

The physical content of our analysis is to interpret the essential role of the VV coefficients in determining magnetic properties, even at low fields and temperatures, where they are often neglected in pseudospin-1/2 models. Quantifying the $\mathrm{VV}$ corrections allows us to demarcate the field-temperature 
range over which a pseudospin-1/2 approximation is appropriate in $\mathrm{CsYbSe}_{2}$, and to describe the high-field limit accurately. The resulting full characterization of the CEF spectra is essential for investigating their experimental consequences, for example, when the application of intense magnetic fields causes multiple real or avoided level crossings in narrowly spaced CEF spectra. It is also a prerequisite to study further mechanisms leading to different forms of magnetic frustration, in which additional degrees of freedom, such as phonons, hybridize with the electronic spectrum to cause profound effects to appear in low-temperature thermodynamics and transport properties [41,42]. Finally, while we have focused on one example material, our analysis can be applied widely to localized $4 f$-electron systems.

The structure of this paper is as follows. In Sec. II, we introduce $\mathrm{CsYbSe}_{2}$, our experimental methods, the complete Stevens operator formalism for CEF levels and some approximate treatments. In Sec. III, we show all the results of our susceptibility measurements and their analysis for the two primary field directions. Section IV presents our RTM measurements and extraction of the magnetotropic coefficients, allowing us to determine the full set of Stevens operators. In Sec. V, we discuss the physical interpretation of the results and their consequences for experimental analysis, effective pseudospin-1/2 models in frustrated quantum magnetism, and materials selection for candidate QSL systems. Section VI contains a brief summary of our contribution and four Appendices provide additional information concerning details of the analysis.

\section{MATERIALS AND METHODS}

While most of the $A \mathrm{Yb} X_{2}$ family crystallizes in the $R \overline{3} \mathrm{bm}$ space group [30], the layer stacking sequence of $\mathrm{CsYbSe}_{2}$ gives a $P 6_{3} / m m c$ structure $[43,44]$, in which the triangular-lattice planes are constructed from edge-sharing

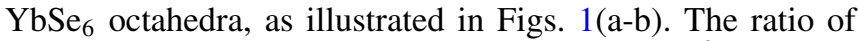
inter- and intra-layer distances separating the $\mathrm{Yb}^{3+}$ ions results in a quasi-2D magnetic system [43]. Within each plane, the Se atoms mediate identical AFM superexchange interactions between all nearest-neighbor $\mathrm{Yb}^{3+}$ ion pairs, resulting in highly frustrated triangular magnetism. In fact the system does not order at zero field for temperatures down to $0.4 \mathrm{~K}$, although an applied in-plane field induces an up-up-down ordering, as demonstrated by the observation of a $1 / 3$ plateau in the magnetization, $M(H)$, at this temperature [43].

Magnetization and susceptibility measurements were performed on single-crystalline samples using a Quantum Design MPMS. All susceptibilities we report are obtained from $\chi_{a b, c}=\frac{d M_{a b, c}}{d H}$, where the indices denote measurements performed with the field oriented in the triangular-lattice plane $(H \| a b)$ or perpendicular to it $(H \| c)$.

Beyond the 7-T upper limit of our magnetization measurements, we employ resonant torsion magnetometry (RTM) to probe the nature of the magnetic anisotropy. The sample is mounted on the tip of a vibrating cantilever and the measured shifts in the resonant frequency $\left(f_{0} \approx 40 \mathrm{kHz}\right)$ reflect changes in the magnetic rigidity caused by changes in the direction of the applied magnetic field, which are quantified by a tensor of magnetotropic coefficients, $k(\vec{H})$. We focus on $k(H, T)$ at the high-symmetry angles $\theta=\pi / 2$ (to measure $k_{a b}$ ) and 0 $\left(k_{c}\right)$, where $\theta$ is the polar angle of the applied field measured from the crystalline $c$ axis. The measurement configuration is summarized in Sec. IV and described in detail elsewhere $[45,46]$. All RTM data were taken using the capacitive magnet of the NHMFL pulsed-field facility at Los Alamos National Laboratory.

\section{A. CEF Analysis and model Hamiltonian}

$\mathrm{Yb}^{3+}$ ions subject to the CEF interactions of their surrounding anion charge distribution have a total $J=7 / 2$ ground-state multiplet of allowed electronic states. Unlike the triangular-lattice material $\mathrm{YbMgGaO}_{4}$, the $\mathrm{Yb}^{3+}$ ions in $\mathrm{CsYbSe}_{2}$ are largely free from any site disorder associated with the nonmagnetic ions. To describe $\mathrm{CsYbSe}_{2}$, we parametrize the single-ion CEF interaction as a linear combination of the six symmetry-allowed Stevens operators, $\hat{O}_{n}^{m}$, for the $D_{3 d}$ site symmetry of the $\mathrm{YbSe}_{6}$ octahedral environment [21,23-26,28-30] to obtain

$\hat{\mathcal{H}}_{\mathrm{CEF}}=B_{2}^{0} \hat{O}_{2}^{0}+B_{4}^{0} \hat{O}_{4}^{0}+B_{4}^{3} \hat{O}_{4}^{3}+B_{6}^{0} \hat{O}_{6}^{0}+B_{6}^{3} \hat{O}_{6}^{3}+B_{6}^{6} \hat{O}_{6}^{6}$.

This Hamiltonian splits the $J=7 / 2$ multiplet into four Kramers doublets, $\left|n_{ \pm}\right\rangle$with $n=0,1,2,3$, whose energies we use to define the separations from the ground-state doublet ( $n=0)$ as $\Delta_{10}, \Delta_{20}$, and $\Delta_{30}$ [Fig. 1(c)]. The corresponding wave functions are obtained by diagonalizing Eq. (1). A CEF spectrum for the zero-field limit can be identified using spectroscopic probes, particularly INS and Raman spectroscopy, but to date little information is available with which to investigate the high-field reorganization of the energy spectrum.

The symmetries of the triangular lattice of $\mathrm{Yb}^{3+}$ ions permit a nearest-neighbor superexchange interaction with $\mathrm{XXZ}$ spin symmetry [47]. Additional symmetry-allowed and bonddependent anisotropic pseudo-dipolar exchange terms [48] are found to give vanishing contributions in a standard Weiss mean-field approximation. Thus we restrict our analysis to the minimal XXZ spin model, $\hat{H}_{\mathrm{XXZ}}$, describing nearest-neighbor interactions between adjacent in-plane $J=7 / 2$ moments in terms of two interactions, $\mathcal{J}_{\perp}$ and $\mathcal{J}_{z}$, which are the respective couplings of spin components transverse and parallel to $\hat{z}$, i.e.,

$$
\hat{\mathcal{H}}_{\mathrm{XXZ}}=\sum_{\langle i j\rangle}\left[\mathcal{J}_{\perp}\left(\hat{J}_{i, x} \hat{J}_{j, x}+\hat{J}_{i, y} \hat{J}_{j, y}\right)+\mathcal{J}_{z} \hat{J}_{i, z} \hat{J}_{j, z}\right],
$$

in which the indices $\langle i, j\rangle$ refer to nearest-neighbor lattice sites and $\hat{J}_{i, \gamma}$, with $\gamma=x, y, z$, labels the components of spin $J=7 / 2$ moments on site $i$.

To account for Zeeman coupling to the external field we add the term

$$
\hat{\mathcal{H}}_{\mathrm{Z}}=-\mu_{0} \mu_{B} g_{J} \mathbf{H} \cdot \sum_{i} \hat{\mathbf{J}}_{i},
$$

where the Landé $g$ factor, $g_{J}=8 / 7$, is used for $\mathrm{Yb}^{3+}$ moments. We note that the quantization axis defining the $\hat{z}$ direction of the chosen basis of spin operators in $\hat{H}_{\mathrm{CEF}}$ and $\hat{H}_{\mathrm{XXZ}}$ is identically the crystallographic $c$ axis, whence the component $H_{z}$ refers to a field $(\mathbf{H} \| c)$ applied along the $c$ axis in experiment. Similarly, $H_{\perp}=\mathbf{H} \| a b$ refers to a field component perpendicular to the $c$ axis, which lies precisely 
in the hexagonally symmetric $a b$ plane. In none of our experiments (magnetization, susceptibility, RTM) did we find a discernible difference in the response for different in-plane field directions, and hence we do not distinguish between these. The two separate contributions to the spin response under an external magnetic field, arising from the single-ion anisotropy and the superexchange anisotropy (both of which have their origin in the CEF spectrum), are then captured by the Hamiltonian

$$
\hat{\mathcal{H}}_{\text {tot }}=\hat{\mathcal{H}}_{\mathrm{CEF}}+\hat{\mathcal{H}}_{\mathrm{XXZ}}+\hat{\mathcal{H}}_{\mathrm{Z}}
$$

\section{B. Weiss mean-field approximation}

We treat the physics of the system at finite temperature within a self-consistent Weiss mean-field approximation for the $\mathrm{Yb}^{3+}$ spins, which we will find captures the bulk magnetic behavior of $\mathrm{CsYbSe}_{2}$ exceptionally well at all measurement temperatures $(T \geqslant 2 \mathrm{~K})$. In the Weiss mean-field treatment, Eq. (4) reduces to a system of $N$ decoupled $\mathrm{Yb}^{3+}$ ions each subject to the Hamiltonian

$$
\begin{aligned}
\hat{\mathcal{H}}_{\mathrm{MF}}^{\mathrm{sg}}= & \hat{\mathcal{H}}_{\mathrm{CEF}}-\frac{1}{2} q\left[\mathcal{J}_{\perp}\left\langle\hat{J}_{x}\right\rangle^{2}+\mathcal{J}_{z}\left\langle\hat{J}_{z}\right\rangle^{2}\right] \\
& -\left[\mu_{0} \mu_{B} g_{J} \mathbf{H}-q\left(\mathcal{J}_{\perp}\left\langle\hat{J}_{x}\right\rangle \hat{\mathbf{x}}+\mathcal{J}_{z}\left\langle\hat{J}_{z}\right\rangle \hat{\mathbf{z}}\right)\right] \cdot \hat{\mathbf{J}},
\end{aligned}
$$

where $q=6$ is the nearest-neighbor coordination number on the triangular lattice and the mean-field expectation values of the spin operators are determined self-consistently as $\left\langle\hat{J}_{\alpha}\right\rangle=\operatorname{Tr}\left[\hat{J}_{\alpha} e^{-\beta \hat{\mathcal{H}}_{\mathrm{MF}}^{\mathrm{sg}}}\right] / Z$, where $Z=\operatorname{Tr}\left[e^{-\beta \hat{\mathcal{H}}_{\mathrm{MF}}^{\mathrm{sg}}}\right]$ and $\beta=$ $1 / k_{B} T$. Because we find that the bulk magnetic susceptibility of $\mathrm{CsYbSe}_{2}$ is almost entirely uniaxial, we make the additional simplifying assumption that the external magnetic field lies in the $x z$ plane, such that $\mathbf{H}=H_{\perp} \hat{\mathbf{x}}+H_{z} \hat{\mathbf{z}}$.

The precise determination of the mean-field Hamiltonian given in Eq. (5) poses a major challenge because it contains eight unknown parameters, six coefficients $\left\{B_{m}^{n}\right\}$ of the Stevens operators in Eq. (1) and two energy scales, $\mathcal{J}_{\perp}$ and $\mathcal{J}_{z}$, specifying the interactions of the $J=7 / 2$ spins. INS spectra are used widely as the starting point for extracting the coefficients $\left\{B_{m}^{n}\right\}[18,19]$, but as noted above measuring the three CEF level splittings, $\Delta_{10}, \Delta_{20}$, and $\Delta_{30}$ [Fig. 1(c)], is not sufficient to determine six unknowns. Electron spin resonance (ESR) [27] probes a much lower frequency range, making it the method of choice for determining effective $g$ factor values in the ground doublet, while the $T$ dependence of the linewidth can be used to estimate $\Delta_{10}[23,25,30]$; in very well characterized systems, the linewidth may also be used to discuss the effective exchange anistropy between $\mathcal{J}_{\perp}$ and $\mathcal{J}_{z}$ [27].

Thus the parameter space for fitting the measured CEF spectra usually remains highly degenerate, even with accurate spectral measurements in an applied field and wellconstrained $g$ tensors, and hence the uniqueness of a fitted set of Stevens operator coefficients cannot be guaranteed $[28,29,37]$. The method we apply to solve this problem has two key components. First we apply a detailed consideration of the second-order corrections in the field dependence of the CEF spectrum, encoded as the VV coefficients we extract from the low-field susceptibility. Then we leverage extensive RTM data providing systematic temperature- and field-dependent information about the magnetotropic coeffi-

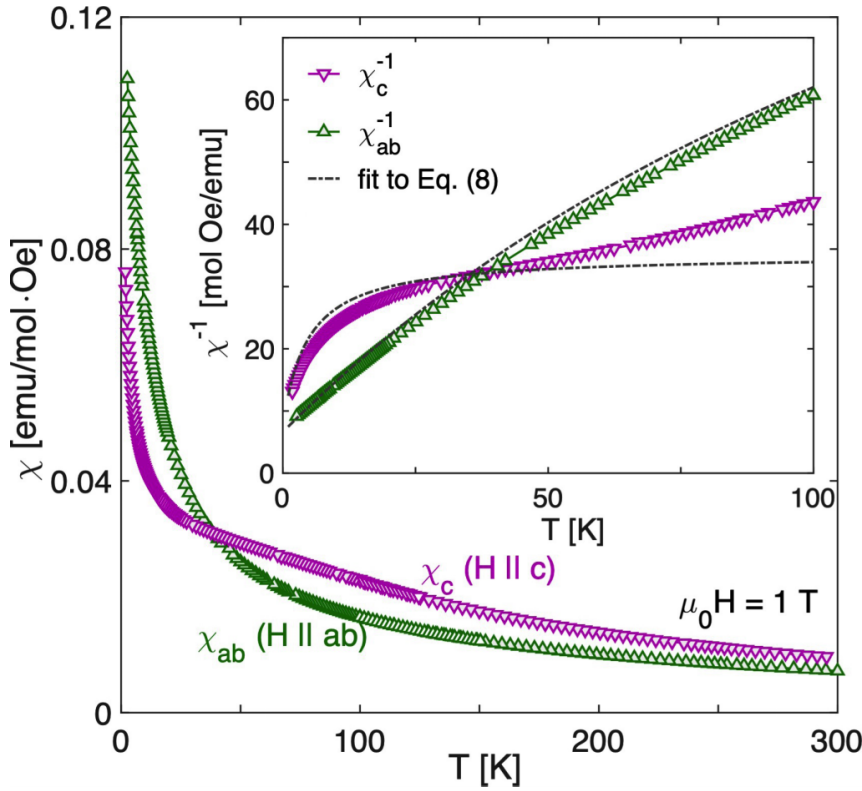

FIG. 2. Temperature dependence of the magnetic susceptibilities, $\chi_{a b}$ and $\chi_{c}$, measured for $\mathrm{CsYbSe}_{2}$ in a field of $\mu_{0} H=1 \mathrm{~T}$ applied respectively in the $a b$ and $c$ directions. The inset shows the inverse susceptibilities, $\chi_{a b}^{-1}$ and $\chi_{c}^{-1}$, compared with fits obtained by applying Eq. (8) in the regime $T \leqslant 45 \mathrm{~K}\left(\ll \Delta_{10} / k_{B}\right)$ in order to determine the interaction parameters, $\mathcal{J}_{\perp}$ and $\mathcal{J}_{z}$, and the $\mathrm{VV}$ coefficients, $\alpha_{\perp}^{0}$ and $\alpha_{z}^{0}$, for the ground-state doublet.

cients for the two high-symmetry field directions to fix a unique set $\left\{B_{m}^{n}\right\}$.

\section{ANISOTROPIC MAGNETIC SUSCEPTIBILITIES}

\section{A. Experiment: deviations from Curie-Weiss}

In Fig. 2, we show the temperature dependence $\mathrm{s}$ of the two magnetic susceptibilities, $\chi_{a b}(T)$ and $\chi_{c}(T)$, measured for $\mathrm{CsYbSe}_{2}$ in the presence of an external magnetic field $\mu_{0} H=$ $1 \mathrm{~T}$ applied respectively within the $a b$ plane and along the $c$ axis. There is no indication of long-range ordering down to $T=2 \mathrm{~K}$. The crossing of the two curves around $T=35 \mathrm{~K}$ reflects a crossover from easy-plane behavior $\left(\Delta \chi=\chi_{a b}-\right.$ $\left.\chi_{c}>0\right)$ at low temperatures to easy-axis anisotropy $(\Delta \chi<0)$ at high $T$.

Both susceptibilities increase rapidly as the temperature is lowered below $50 \mathrm{~K}$, and the corresponding inverse quantities shown in the inset of Fig. 2 make clear the susceptibility anisotropy, with $\chi_{c}^{-1}(T)$ exhibiting a significantly sharper downward trend as $T$ decreases. While a qualitative inspection suggests that a Curie-Weiss form might capture $\chi_{a b}^{-1}(T)$ for $T<50 \mathrm{~K}$, this is clearly impossible for $\chi_{c}^{-1}(T)$. This type of low- $T$ downturn in $\chi^{-1}(T)$ is observed quite commonly in similar rare-earth magnetic materials $[16,20,23,25,40,49]$, but to date lacks a detailed analysis. Next we show that this behavior can be explained entirely by analyzing the field-induced evolution of the CEF energy levels, where the leading deviations from a linear (effective Zeeman) form are contained in two strongly anisotropic ground-state VV coefficients. 


\section{B. Van Vleck coefficients and low-field limit}

The splitting of the CEF levels in a finite applied magnetic field can be understood systematically by perturbation theory. We express the $H$-linear and -quadratic corrections to the energy eigenvalues of $\hat{\mathcal{H}}_{\mathrm{CEF}}$ due to $\hat{H}_{\mathrm{Z}}$ in the form

$$
\begin{gathered}
E_{n, \pm}(H)=E_{n}^{0} \pm \frac{1}{2} \mu_{0} \mu_{B} \sqrt{\left(g_{\perp}^{n} H_{\perp}\right)^{2}+\left(g_{z}^{n} H_{z}\right)^{2}} \\
+\alpha_{\perp}^{n} H_{\perp}^{2}+\alpha_{z}^{n} H_{z}^{2}+\mathcal{O}\left(H^{3}\right),
\end{gathered}
$$

where $E_{n}^{0}$ refers to the four CEF energy levels at zero field (and setting the ground-state energy, $E_{0}^{0}$, to zero gives $E_{n}^{0}=$ $\Delta_{n 0}$ for $n=1,2$, and 3). The second term describes the $H$-linear Zeeman splitting with a generalized $g$-tensor for all levels $(n=0,1,2,3)$ defined as $g_{\perp(z)}^{n}=2 g_{J}\left\langle n_{ \pm}\left|\hat{J}_{x(z)}\right| n_{\mp( \pm)}\right\rangle$, where the subscripts $\perp, z$ denote a field applied respectively within the $a b$-plane or along the $c$-axis. The conventional effective spin-1/2 $g$-tensor components are $g_{\perp}^{0}$ and $g_{z}^{0}$, to which we refer henceforth, without the superscript 0 , as the $g$ factors of the system.

We define all of the phenomena obtained at second order in the perturbative effect of $\hat{H}_{\mathrm{Z}}$ on the zero-field eigenstates of $\hat{H}_{\mathrm{CEF}}$ as the $\mathrm{VV}$ contribution. To describe the full fieldand temperature dependence of the susceptibility we define the VV coefficients, $\alpha_{\perp}^{n}$ and $\alpha_{z}^{n}$, for the $n$th CEF level by

$$
\alpha_{\perp(z)}^{n}=\left(\mu_{0} \mu_{B} g_{J}\right)^{2} \sum_{n^{\prime} \neq n} \frac{\left|\left\langle n_{ \pm}^{\prime}\left|\hat{J}_{x(z)}\right| n_{+}\right\rangle\right|^{2}}{\Delta_{n n^{\prime}}},
$$

where $\Delta_{n n^{\prime}}=E_{n}^{0}-E_{n^{\prime}}^{0}$. Although it is often stated that one may define a "VV susceptibility," $\chi_{\mathrm{VV}}$, that is a small, additive, temperature-independent, and paramagnetic contribution to the total susceptibility, this is rarely an accurate approximation. By inspecting the form of the VV coefficients, one observes that the $n=0$ terms should be negative, giving the expected type of second-order correction to the ground state. The physics content of the anisotropic VV coefficients can be read from Fig. 1(c), where the field dependence of the CEF levels is quite strongly nonlinear due to level-repulsion effects between adjacent doublets. In $\mathrm{CsYbSe}_{2}$ this repulsion, which is equivalent to a negative curvature of the lower (and positive of the upper) branch in each case, is strongest between the $n=0$ and 1 doublets for $H \| c$.

We obtain analytical formulas for the low-field magnetic susceptibilities of the system by using the $N$-particle partition function calculated with Eq. (5). The full expression for $\chi_{a b(c)}(T)$, which includes the contributions of all four CEF levels, is presented in Eq. (A1) of Appendix A. Here we focus on the regime of temperatures sufficiently small that only the lowest-lying Kramers doublet need be considered, i.e., $k_{B} T \ll \Delta_{10}$, and write the inverse susceptibility as

$$
\begin{aligned}
\chi_{a b(c)}^{-1} & =\frac{1}{\mathcal{C}}\left[\frac{T}{\left|\left\langle 0_{+}\left|\hat{J}_{x(z)}\right| 0_{-(+)}\right\rangle\right|^{2}-\frac{2 k_{B} \alpha_{\perp(z)}^{0}}{\mu_{0}^{2} \mu_{B}^{2} g_{J}^{2}} T}+\Theta_{\perp(z)}^{\mathrm{CW}}\right] \\
& =\frac{\mu_{0} k_{B}}{N}\left[\frac{T}{\frac{1}{4} g_{\perp(z)}^{2} \mu_{0}^{2} \mu_{B}^{2}-2 \alpha_{\perp(z)}^{0} k_{B} T}+\Theta_{\perp(z)}^{\mathrm{CW}}\right]
\end{aligned}
$$

where $\mathcal{C}=N \mu_{0} \mu_{B}^{2} g_{J}^{2} / k_{B}$ is a constant. We have defined the quantities $\Theta_{\perp(z)}^{\mathrm{CW}}=q \mathcal{J}_{\perp(z)} / k_{B}$ in order to obtain an adapted
Curie-Weiss $(\mathrm{CW})$ form for the two applied-field directions and in the second line we have used the definition of the $g$ factors (above) to make this form more transparent. We note that the regime of validity of Eq. (8) is also that in which the system can be approximated by an effective pseudospin-1/2 description.

It is clear from Eq. (8) that the contrast to a conventional CW form, $\chi^{-1} \propto T+\widetilde{\Theta}$, is the additional $T$-linear term in the denominator, whose prefactor is the corresponding VV coefficient. An explicit comparison is presented in Appendix B. The key advantage of our formulation is to observe that the same (VV) coefficients describing the $H^{2}$ correction that gives the leading nonlinear contribution to the CEF levels at low temperatures and finite fields [Eq. (6)] are those describing the nonlinear, "beyond-CW" form of the susceptibility at zero field and finite temperatures [Eq. (8)]. Thus one may conclude that the latter effect is also related to level-repulsion between doublets, the fact that the susceptibility is a second field derivative of the free energy meaning that second-order perturbative effects do not vanish in the limit $H \rightarrow 0$.

Returning to Fig. 2, the gray dashed lines in the inset show fits to Eq. (8), made in the regime $T<45 \mathrm{~K}$, for each field direction. From these fits we obtain two exchange energies, $\mathcal{J}_{\perp}$ and $\mathcal{J}_{z}$ in Eqs. (2) and (5), and two VV coefficients for the ground-state doublet. A complete fit is deferred to Sec. V. Starting with the VV coefficients, we find the values $\alpha_{\perp}^{0}=$ $-(2.9 \pm 0.1) \times 10^{-4} \mathrm{meV} / \mathrm{T}^{2}$ from $\chi_{a b}^{-1}$ and $\alpha_{z}^{0}=-(17.9 \pm$ $0.1) \times 10^{-4} \mathrm{meV} / \mathrm{T}^{2}$ from $\chi_{c}^{-1}$. We stress that $\alpha_{z}^{0}$ is six times larger than $\alpha_{\perp}^{0}$, which for all fields beyond $5 \mathrm{~T}$ becomes clearly manifest as a much larger downward level-repulsion of the lowest Kramers doublet [Fig. 1(c) and the quantitative analysis of Sec. V]. Turning to the magnetic interactions, we find $\mathcal{J}_{\perp}=0.54 \pm 0.01 \mathrm{~K}$ from $\chi_{a b}$ and $\mathcal{J}_{z}=0.61 \pm 0.01 \mathrm{~K}$ from $\chi_{c}$, both encapsulated in $\Theta_{\perp(z)}^{\mathrm{CW}}$. If one reduces the system to an effective pseudospin- $1 / 2$ model, the corresponding interaction terms are $\mathcal{J}_{\perp}^{\prime}=5.12 \mathrm{~K}(\simeq 0.44 \mathrm{meV})$ and $\mathcal{J}_{z}^{\prime}=$ $0.84 \mathrm{~K}(\simeq 0.07 \mathrm{meV})$, as detailed in Appendix C. We discuss the physical implications of these interaction parameters in Sec. V B.

As noted above, downward curvature of the lowtemperature $\chi^{-1}$ has been observed in other rare-earth compounds and our fitting results demonstrate that this feature should be characterized by using the VV coefficients as a part of a full description of the anisotropic magnetism. The validity of Eq. (8) as a replacement for the CW form of the susceptibility is confirmed by capturing the different $T$ dependencies correctly for the two field directions with two VV coefficients that are consistent with Eq. (6). Although the $\mathrm{VV}$ coefficients are not parameters appearing directly in the system Hamiltonian, they impose constraints that are essential for a unique determination of the full parameter set, a topic we discuss further in the next section.

In Table I, we compare the VV coefficients and other physical characteristics for a variety of $\mathrm{Yb}$ delafossites. For all compounds listed other than $\mathrm{CsYbSe}_{2}$, the $\Delta_{10}$ and $g$ tensor parameters are taken from experimental data. All $\alpha_{\perp}^{0}$ and $\alpha_{z}^{0}$ values were calculated from Eq. (7) using the Stevens coefficients $\left(B_{m}^{n}\right)$ provided by each reference, where they were obtained from fits to the CEF spectrum obtained by INS. We stress again the fact that, in several of the studies cited, 
TABLE I. Comparison of the lowest zero-field CEF level splitting, $\Delta_{10}, g$-tensor components, and the VV coefficients for $n=0$ in a number of Yb-based triangular-lattice compounds. For $\mathrm{CsYbSe}_{2}$ we show two sets of $g$ factors and VV coefficients, one obtained by a full calculation [Eq. (7)] using the $B_{m}^{n}$ coefficients and wave functions, i.e., assisted by fitting to the RTM data as shown in Sec. IV, and the other from fitting $\chi_{a b, c}(T)$ using Eq. (8). For the other compounds, $\Delta_{10}$ and the $g$ factors are quoted from the respective references and we calculated the VV coefficients using the reported $B_{m}^{n}$ coefficients. The compound nominally most similar to CsYbSe $\mathrm{C}_{2}, \mathrm{NaYbSe}_{2}$, displays slightly less anisotropy in its VV coefficients and this is consistent with the differing forms of $\chi_{a b}(T)$ and $\chi_{c}(T)$ reported in Ref. [25]. For NaYbO $\mathrm{N}_{2}$ we note that the two sets of Stevens coefficients (fits 1 and 2) deduced in Ref. [28] yield dramatically different values of $\alpha_{\perp}^{0}$ and $\alpha_{z}^{0}$, which underlines the crucial role of the $\mathrm{VV}$ coefficients in a complete and consistent characterization of $\mathcal{H}_{\mathrm{CEF}} . \mathrm{YbMgGaO}_{4}$ is found to be least anisotropic among these materials, and its small VV coefficients are consistent with the reported Curie-Weiss form of the susceptibility.

\begin{tabular}{lcccccc}
\hline \hline & $\Delta_{10}(\mathrm{meV})$ & $g_{\perp}$ & $g_{z}$ & $\alpha_{\perp}^{0}\left(10^{-4} \frac{\mathrm{meV}}{\mathrm{T}^{2}}\right)$ & $\alpha_{z}^{0}\left(10^{-4} \frac{\mathrm{meV}}{\mathrm{T}^{2}}\right)$ & Reference \\
\hline $\mathrm{CsYbSe}_{2}$ & 13.6 & 3.52 & 1.33 & -3.31 & -18.8 & calculated from Eq. (5) \\
$\mathrm{CsYbSe}_{2}$ & - & 3.77 & 1.76 & -2.93 & -17.9 & $\chi_{a b, c}(T)$ fit to Eq. (8) [Fig. 2] \\
$\mathrm{NaYbSe}_{2}$ & $17.5 / 17.7$ & $2.87 / 2.87$ & $1.18 / 1.33$ & $-5.51 /-5.44$ & $-12.5 /-18.8$ & fit 1/fit 2 in Ref. [29] \\
$\mathrm{NaYbSe}_{2}$ & 13.8 & 3.13 & 1.01 & - & - & ESR [25] \\
$\mathrm{NaYbO}_{2}$ & $34.0 / 34.7$ & $3.39 / 3.54$ & $1.71 / 1.75$ & $-1.10 /-2.85$ & $-7.39 /-3.62$ & fit 1/fit 2 in Ref. [28] \\
$\mathrm{NaYbS}_{2}$ & 16.7 & 3.19 & 0.57 & - & - & ESR [23] \\
$\mathrm{YbMgGaO}_{4}$ & $39.3 / 39.4$ & $3.22 / 3.21$ & $3.70 / 3.73$ & $-2.25 /-2.29$ & $-2.60 /-2.58$ & fit 1/fit 2 in Ref. [37] \\
\hline \hline
\end{tabular}

different sets of Stevens coefficients can provide equally good descriptions ("fit 1" and "fit 2") of the same INS data due to the underconstrained nature of the problem. The anisotropy of $\mathrm{CsYbSe}_{2}, \alpha_{z}^{0} / \alpha_{\perp}^{0} \approx 6$, is strikingly higher than the values reported for other compounds in the same family. Although all of the $\mathrm{NaYbX}_{2}$ materials seem to show considerable directional anisotropy [30], this may be less severe for $\mathrm{NaYbO}_{2}$, except that the two sets of proposed $B_{m}^{n}$ parameters yield wildly different VV coefficients, indicative of an underlying ambiguity of the type we demonstrate how to resolve. In this regard, the sole nondelafossite in Table $\mathrm{I}, \mathrm{YbMgGaO}_{4}$, is a nearly isotropic outlier.

Before proceeding, we reiterate two important attributes of the VV coefficients as a mean of characterizing the magnetic anisotropy of a CEF system. First, nonzero VV coefficients are immediately evident in $\chi(T)$, as strong deviations from a CW form, and thus failure to account for them means that the interaction parameters, $\mathcal{J}_{\perp, z}$, cannot be determined correctly. Second, it is evident from Eq. (8) that caution is required in applying an effective pseudospin-1/2 description, because even at low $T$, where only the lowest Kramers doublet is thermally populated, the repulsion from the higher CEF levels cannot be ignored. Hence the twin roles of the anisotropic VV coefficients in dictating $H$ - and $T$-dependent physical properties generic to many $4 f$ electronic systems (Table I) must be taken into account to obtain a meaningful description of the spin physics.

\section{RESONANT TORSION MAGNETOMETRY}

\section{A. Dependence on field and temperature}

Having characterized the magnetic response at low fields using $\chi(T)$, and thereby obtained four independent physical quantities to include in the fitting procedure, we turn for more information to the magnetropic coefficients. The RTM method allows these to be measured over wide ranges of both field and temperature, which we will show provides enough input for an unambiguous determination of all the remaining free parameters in Eq. (5). The magnetotropic coefficient is defined as $k(H, \vartheta)=\partial^{2} F(H, \vartheta) / \partial \vartheta^{2}$, where $F(H, \vartheta)$ is the portion of the Helmholtz free energy depending on the magnitude and orientation of the magnetic field $(\vartheta$ is the angular direction of $\hat{H}$ measured in the plane of vibration of the sample $[45,46]) . k$ quantifies the magnetic rigidity of a material, whose origin lies in the energy cost of rotating a sample with an anisotropic magnetic free energy in a finite field, and RTM measurements constitute a highly sensitive probe of this magnetoanisotropy.

The variation of the magnetotropic coefficients at finite field produces a shift in the resonant frequency of a system composed of a cantilever and an attached sample given by

$$
\frac{\Delta f}{f_{0}}=\frac{k}{2 K}
$$

with $K$ the intrinsic bending stiffness of the cantilever at zero field $[45,46]$. In the low-field limit, where $\chi$ is constant at constant temperature, both the torque and the magnetotropic coefficient are not only straightforward functions of the polar angle, $\theta$, but are also quadratic in $H$. Thus the magnetotropic coefficient can be expressed in terms of the difference, $\Delta \chi=$ $\chi_{a b}-\chi_{c}$, between the principal components of the susceptibility tensor in the plane of vibration, as $k=\Delta \chi H^{2} \cos 2 \theta$. More detailed derivations of field-dependent expressions for the high-symmetry angles $\left(k_{a b}\right.$ for $\theta=0$ and $k_{c}$ for $\left.\theta=\pi / 2\right)$ can be deduced by introducing the transverse susceptibility, as shown in Appendix D.

Figures 3(a) and 3(b) show the frequency shifts, $\Delta f_{a b}$ and $\Delta f_{c}$, which are directly proportional to the magnetotropic coefficients, $k_{a b}$ and $k_{c}$ [Eq. (9)], as functions of field in the $H \| a b$ and $H \| c$ geometries. We note that the vibration plane of the cantilever and the rotation plane of $H$ are the same, meaning that in this experimental configuration the angle $\vartheta$ of Eq. (D2) is identically the polar angle, $\theta$, relative to the crystallographic $c$ axis.

At low fields, where $\chi_{a b}$ and $\chi_{c}$ are $H$-independent constants (i.e., the magnetizations $m_{a b}$ and $m_{c}$ are linear in $H$ ), both $k_{a b}$ and $k_{c}$ are indeed proportional both to $H^{2}$ and to $\Delta \chi$ with opposing signs. As the temperature is increased, 

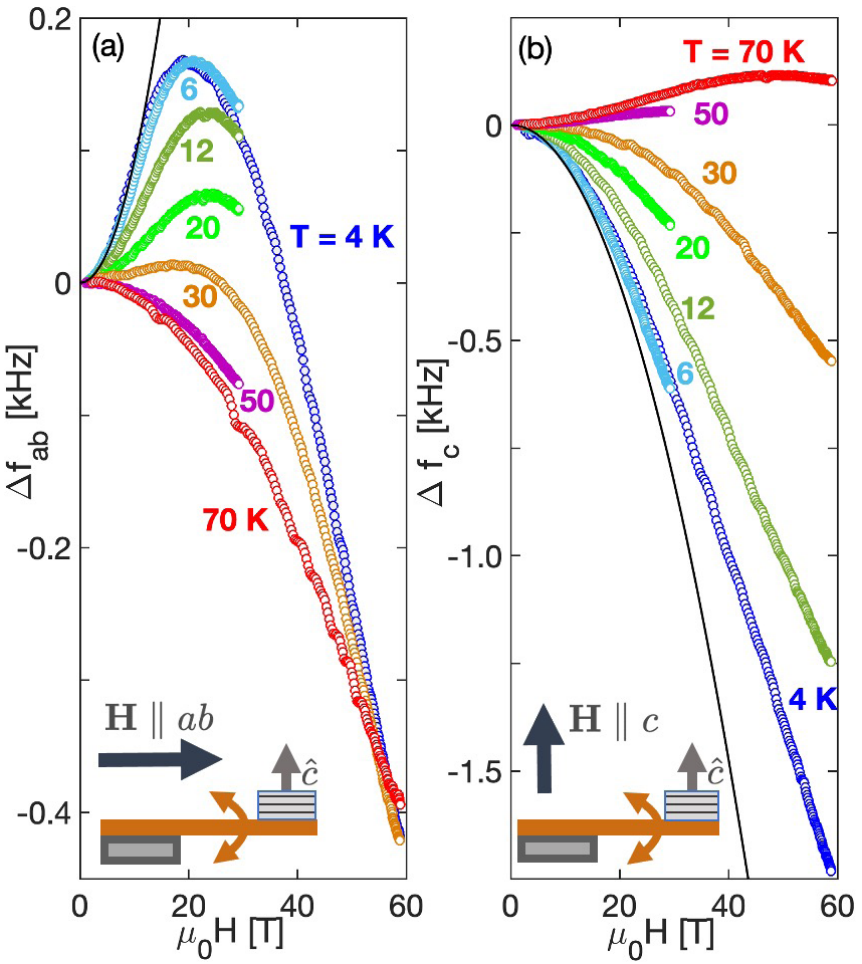

FIG. 3. Dependence on the applied field magnitude of the resonant frequency shifts (a) $\Delta f_{a b}$ for $H \| a b(\theta=\pi / 2)$ and (b) $\Delta f_{c}$ for $H \| c(\theta=0)$. Thin solid lines show the low-field $H^{2}$ dependencies, with coefficients of $\Delta \chi$ and $-\Delta \chi$, taken at $T=4 \mathrm{~K}$ from Fig. 2 . The nonmonotonic $H$ dependence leading to a local maximum in the $H \|$ $a b$ configuration is attributed to the saturation of the magnetization and is explained in Sec. V by considering the effective susceptibility in the transverse direction. The insets illustrate the applied-field configurations of the RTM measurements on the layered triangular lattice of $\mathrm{CsYbSe}_{2}$ in each case.

$\Delta \chi(T)$ in Fig. 2 changes its sign above $50 \mathrm{~K}$, and this is reflected in the sign-changes of $\Delta f_{a b}$ and $\Delta f_{c}$ between $T=30$ and $50 \mathrm{~K}$.

As the field is increased, $\Delta f_{a b}(H)$ deviates from an $H^{2}$ dependence and becomes nonmonotonic, with a local maximum at low temperatures $[T \leqslant 30 \mathrm{~K}$ in Fig. 3(a)]. The origin of this behavior lies primarily in $m_{a b}(H)$ saturating in this field range, which we confirm from our calculations in Sec. V. By contrast, the behavior of $\Delta f_{c}(H)$ remains monotonic in the same $T$ range, where $m_{c}(H)$ continues to increase with $H$. We comment that $\Delta f_{c}(H)$ does exhibit a weak maximum in $H$ at $T=70 \mathrm{~K}$, whereas no such behavior is found in $\Delta f_{a b}$ at this temperature. This feature is also captured qualitatively by calculating the eigenstates of the full mean-field Hamiltonian [Eq. (5)], as we show next.

\section{B. Fitting $\boldsymbol{k}_{a b}$ and $\boldsymbol{k}_{c}$}

Taking the magnetic interaction parameters $\mathcal{J}_{\perp}$ and $\mathcal{J}_{z}$ determined from $\chi_{a b, c}(T)$ (Fig. 2), we fit the measured data for $k_{a b}(H)$ and $k_{c}(H)$ using the full mean-field Hamiltonian based on Eq. (5), in which the six coefficients of the Stevens operators are free parameters to be determined. However,
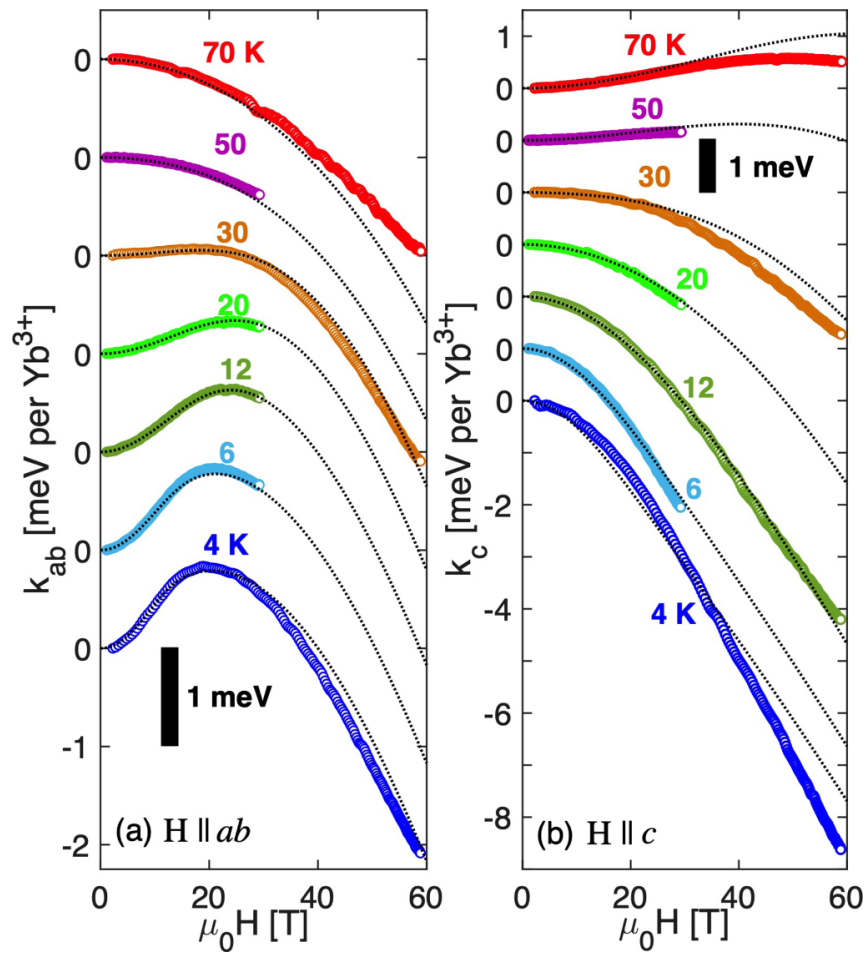

FIG. 4. Magnetotropic coefficients, $k_{a b}$ and $k_{c}$, shown per $\mathrm{Yb}^{3+}$ ion as a function of field in the respective geometries $H \| a b$ (a) and $H \| c$ (b). Open symbols mark measured data and dotted lines show the best fits achieved by self-consistent diagonalization of the full mean-field Hamiltonian [Eq. (5)]. The values of $\mathcal{J}_{\perp}$ and $\mathcal{J}_{z}$ are taken from the susceptibility fits [Fig. 5], leaving the six coefficients of the Stevens operators as free parameters constrained by the extracted VV coefficients, $\alpha_{\perp}^{0}$, and $\alpha_{z}^{0}$. Curves are shown for clarity with a constant offset.

obtaining the ground-state VV coefficients, $\alpha_{\perp}^{0}$ and $\alpha_{z}^{0}$, from the fit to Eq. (8) reduces the number of fitting parameters to four. Given the wide ranges of $T$ and $H$ covered by the RTM data, the remaining unknowns can be determined with an unprecedentedly high level of confidence by using $k_{a b}$ and $k_{c}$.

Figure 4 displays the magnetotropic coefficients converted from the measured $\Delta f$ data in both geometries. It is clear that the fits capture the full field dependence of $k_{a b}(H)$ and $k_{c}(H)$ in an excellent manner, despite the minimal spin model and the mean-field approximation. In particular, the low-field $H^{2}$ curvature is fully consistent with $|\Delta \chi|$ (Sec. IV A). At $\mu_{0} H>$ $30 \mathrm{~T}$, beyond the range of some of the data, the agreement is no longer quantitative for all temperatures simultaneously, but the model continues to capture the majority of the fielddependent behavior for both field directions. One possible source of these deviations would be high-field magnetostrictive effects, which can distort the lattice structure and thus

TABLE II. Values of the Stevens operator coefficients [Eq. (1)] obtained from the fits shown in Fig. 4.

\begin{tabular}{lcccccc}
\hline \hline unit & $B_{2}^{0}$ & $B_{4}^{0}$ & $B_{4}^{3}$ & $B_{6}^{0}$ & $B_{6}^{3}$ & $B_{6}^{6}$ \\
\hline $10^{-2} \mathrm{meV}$ & -42.33 & 1.17 & 54.94 & 0.03 & 0.52 & -0.04 \\
\hline \hline
\end{tabular}




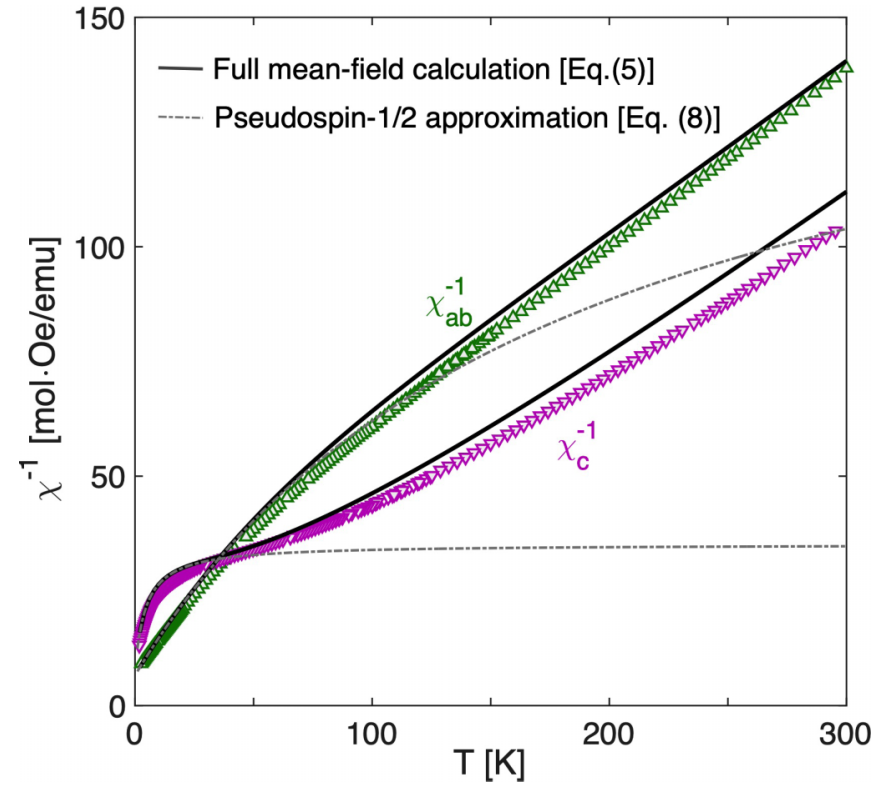

FIG. 5. Data for the measured inverse susceptibilities (symbols, inset Fig. 2) shown over the full range of temperature together with fits (solid lines) computed using the Stevens operator coefficients of Table II. Thin dot-dashed lines show fits obtained from a pseudospin$1 / 2$ description [Eq. (8)] applied at low temperatures, which align well with the full matrix calculation for $T<60 \mathrm{~K}$.

modify both the magnetic interactions and possibly even the sizes of the CEF coefficients at sufficiently large applied fields [50]. We use the optimal fits to obtain the six Stevens operator coefficients [Eq. (1)] shown in Table II and we discuss the physical implications of having this fully determined spin Hamiltonian [Eq. (5)] in the next section.

\section{DISCUSSION}

\section{A. CEF spectrum and VV coefficients}

Having determined a full set of eight fitting parameters from the RTM data, we first verify the self-consistency of this fit against the measured magnetic susceptibilities, which are shown as $\chi_{a b}^{-1}(T)$ and $\chi_{c}^{-1}(T)$ over the full temperature range in Fig. 5. The solid lines show the same quantities calculated from the mean-field Hamiltonian of Eq. (5). The agreement of the fit with the data is quantitatively excellent over the entire measured $T$ range, including temperatures allowing significant population of the higher CEF levels. The dot-dashed lines were calculated using the pseudospin- $1 / 2$ approximation, i.e. the contributions from the ground-state doublet [Eq. (8)], which as expected captures the $T$ dependence below a specific energetic cutoff; by inspection we find this cutoff at $T \approx 60 \mathrm{~K}$ in $\mathrm{CsYbSe}_{2}$ for both directions of $\hat{H}$.

As discussed in Sec. III, the difference in $T$ dependence for the two primary field directions is accounted for in the modeling procedure by the large discrepancy in the VV coefficients. While this anisotropy is also reflected in the very different prefactors of the $H$-quadratic part of the energy spectrum for the two different field geometries, we stress again the fact that it affects the susceptibility strongly even at zero field. As a consequence, the susceptibility fits in Fig. 2 provide an inde-

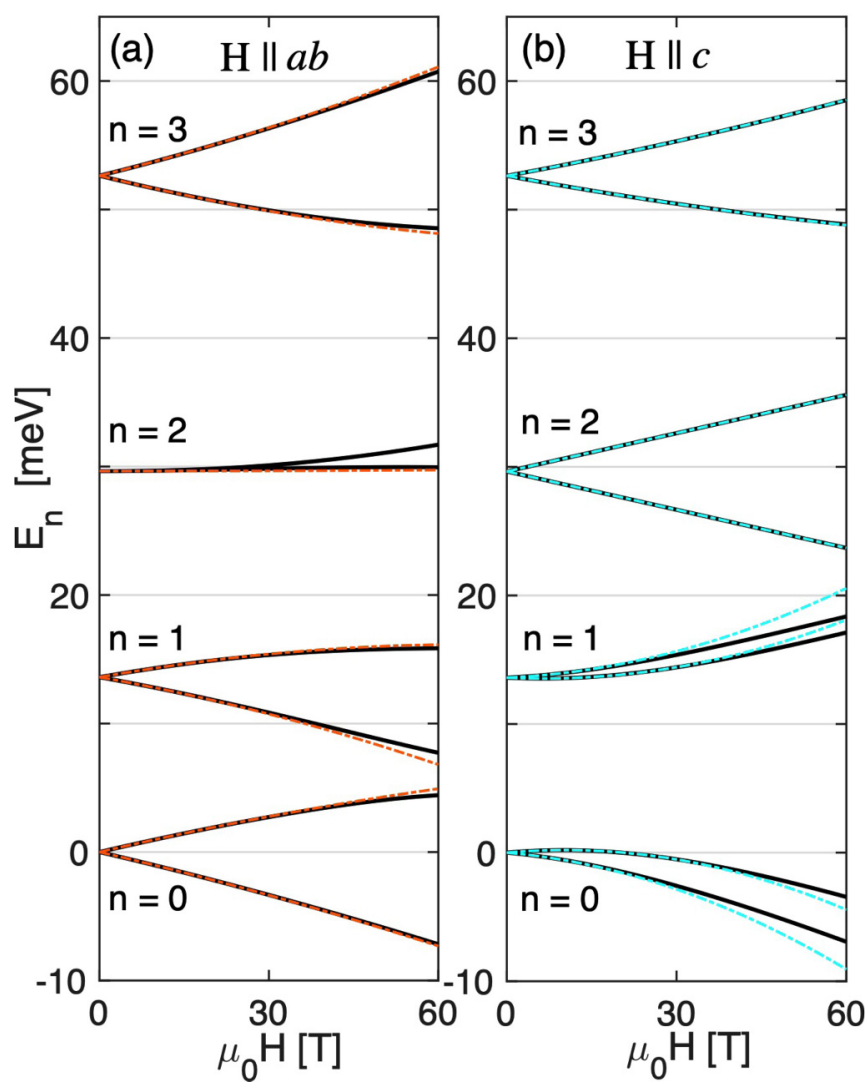

FIG. 6. CEF spectrum calculated using the fitting result from Fig. 4 (solid lines). Quadratic and higher-order dependence on the applied field are clearly visible for all four Zeeman-split Kramers doublets. We comment that the $n=2$ level remains mostly degenerate in fields $\mathbf{H} \| a b$ because of their dipolar-octupolar nature. Dot-dashed lines show the perturbative expression of each energy level at second order, as in Eq. (6).

pendent determination of $\alpha_{\perp}^{0}$ and $\alpha_{z}^{0}$, as well as of the squared matrix elements $\left|\left\langle\left|0_{ \pm}\right| \hat{J}_{x(z)} \mid 0_{\mp( \pm)}\right\rangle\right|^{2}$. These constitute additional constraints on the allowed Stevens operator coefficients that are crucial for reducing the enormous degeneracy of the six-variable parameter space describing the CEF levels. It is well known that suitable sets of coefficients are often highly degenerate, in the sense of yielding many indistinguishable fits of INS data for the CEF spectrum and $g$-tensor values $[18,28,37]$. In Table I, we compare the ground-state $g$-tensor values and VV coefficients obtained by fitting $k_{a b, c}(H)$ (top line, Fig. 4) and $\chi^{-1}$ (second line, Fig. 5), where we find agreement at the $10 \%$ level.

Next we use our fit coefficients to calculate the CEF energy levels, which are shown in Fig. 6 as a function of field at zero temperature. The black solid lines show the spectra calculated, for $\hat{H}$ oriented in the $a b$-plane [panel (a)] and along the $c$-axis [panel (b)], by the direct diagonalization of $\hat{\mathcal{H}}_{\mathrm{CEF}}+\hat{\mathcal{H}}_{\mathrm{Z}}$. Focusing first on zero field, we obtain the level-splittings $\Delta_{10}=$ $13.6 \mathrm{meV}, \Delta_{20}=29.6 \mathrm{meV}$, and $\Delta_{30}=52.6 \mathrm{meV}$. Thus $\Delta_{10}$ in $\mathrm{CsYbSe}_{2}$ is similar to that of $\mathrm{NaYbSe}_{2}(\approx 17.7 \mathrm{meV}$ [29]), but is significantly lower than the values reported for delafossites in which the $\mathrm{Yb}^{3+}$ ion has an oxygen environment $[28,37]$. The $g$-factor values we obtain for the ground state, 
$g_{a b}=3.52$ and $g_{c}=1.33$, are comparable to those measured in all of the Yb-based delafossites [30], as summarized in Table I.

Turning to finite fields, a comparison of the ground-state doublet $(n=0)$ in Figs. 6(a) and 6(b) shows the expected strong anisotropy, which lies predominantly in the fact that the $H$-quadratic component is much larger for $H \| c$ (the aforementioned factor- 6 difference between $\alpha_{z}^{0}$ and $\left.\alpha_{\perp}^{0}\right)$. Because the quadratic curvatures are a consequence of repulsion between adjacent CEF levels, the $n=1$ doublet exhibits a clear opposing curvature for $H \| c$ (dominating the behavior of both doublet components), whereas in the $H \| a b$ configuration this effect is weaker than the higher-order corrections. The dot-dashed lines in Fig. 6 illustrate the fidelity of a fit made only at the level of second-order energy corrections for each $n$ [Eq. (6)], i.e., by using all the VV coefficients, which agrees well until $\mu_{0} H>50 \mathrm{~T}$ in both $\hat{H}$ directions.

Moving up in the CEF spectrum, our results also capture the unique properties of the $n=2$ state. This dipolaroctupolar doublet, $\left|J, m_{J}= \pm 3 / 2\right\rangle[20,49,51,52]$, does not transform as a magnetic dipole because the threefold rotational symmetry excludes any mixing between states with $m_{J}= \pm 3 / 2$ and those with other values of $m_{J}$ [53]. Instead this doublet combines the features of dipolar and octupolar moments [20,49]. In particular, only one component of the dipole moment (in this case oriented along the $c$ axis) appears in the vector of pseudospin- $1 / 2$ operators, which makes the Zeeman splitting of the $n=2$ level appear very different between $H \| a b$ and $H \| c$. In the latter case, the $m_{J}=3 / 2$ state remains an exact eigenstate up to arbitrarily large field, so the Zeeman splitting is exactly $H$-linear with no higher-order perturbative corrections [Fig. 6(b)], i.e., $\alpha_{z}^{2}=0$. By contrast, for $H \| a b$ the matrix elements $\left\langle 2_{ \pm}\left|J_{x}\right| 2_{\mp}\right\rangle$ are zero and the $H$-linear Zeeman splitting vanishes $\left(g_{\perp}^{2}=0\right)$. Thus these doublet components remain nearly degenerate [Fig. 6(a)] until the field is sufficiently strong that higher-order perturbative corrections become visible.

A further key experimental quantity we consider is the magnetization. The solid lines in Fig. 7 show the magnetization of the system at several different temperatures calculated for fields in the two primary directions using the full Hamiltonian matrix [Eq. (5)]. In the low-field range, $m_{a b}$ and $m_{c}$ reproduce exactly our experimental data measured up to $\mu_{0} H=7 \mathrm{~T}$. At higher fields, $m_{a b}$ calculated from the full spectrum changes slope at $\mu_{0} H \approx 12 \mathrm{~T}$ (at $T=4 \mathrm{~K}$ ), beyond which it continues to increase more slowly with increasing field. This hint of saturation behavior is consistent with the broad maximum in the RTM frequency shift, $\Delta f_{a b}(H)$, at low temperatures (Fig. 3). No such behavior is found in $m_{c}$, although the overall slope does decrease as $H$ is raised beyond approximately $25 \mathrm{~T}$, and nor is it immediately evident in the RTM measurements; however, it can be found within the detailed $H$ dependence of the free energy, which we parametrize using the transverse susceptibilities, $\chi_{a b}^{T}(T)$ and $\chi_{c}^{T}(T)$, in Appendix D.

Finally, the magnetization presents an excellent test case for the validity of a pseudospin-1/2 description of $\mathrm{CsYbSe}_{2}$, meaning the extent to which any physical property is explained by the contributions from the ground-state doublet $(n=0)$ alone. In Figs. 7(a) and 7(b), we show in addition

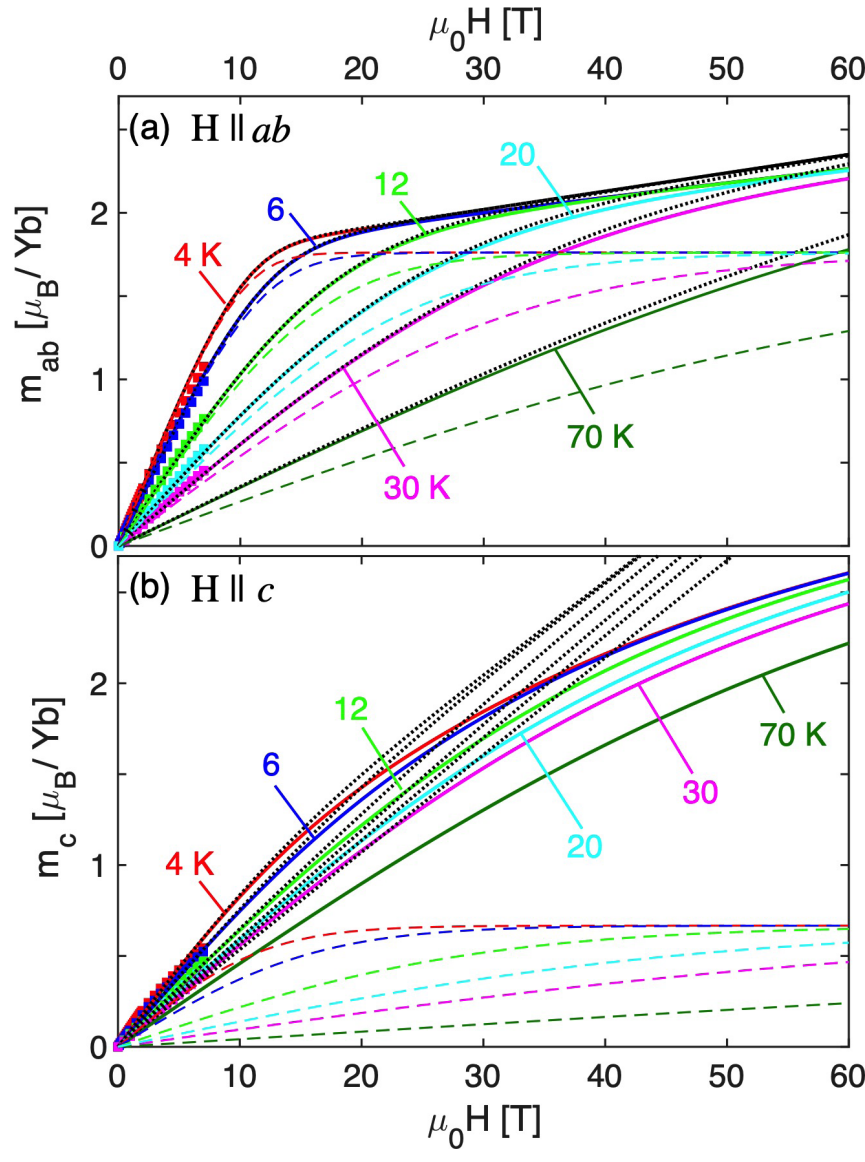

FIG. 7. Magnetizations, $m_{a b}$ (a) and $m_{c}$ (b), calculated for selected values of the temperature from Eq. (5) (solid lines), in a pseudospin-1/2 approximation with VV corrections (dotted lines), and in a pseudospin-1/2 approximation without VV corrections (dashed lines). Solid symbols show magnetization data measured up to $7 \mathrm{~T}$.

the magnetizations obtained using a pseudospin-1/2 approximation with (dotted lines) and without (dashed lines) the VV correction. For reference, the efficacy of the VV corrections in reproducing the full field-induced evolution of the ground doublet can be gauged in Fig. 6. For $m_{a b}(H)$, a pseudospin- $1 / 2$ model with no VV correction captures the field dependence only up to $\mu_{0} H \approx 10 \mathrm{~T}$ before saturating to the generic tanh function of a Zeeman doublet. By contrast, a model with VV correction follows the full solution very closely over the entire field range to $60 \mathrm{~T}$. This contrasts with the situation for $m_{c}(H)$, where even the VV-corrected model shows a clear departure from the full solution that sets in around $15 \mathrm{~T}$, beyond which the approximate treatment separates systematically, and it is clear that higher-order corrections to the doublet spectrum are required. For the model with no $\mathrm{VV}$ correction, $m_{c}(H)$ does not even reproduce the low-field regime. Recalling the very significant magnetic anisotropy $\left(\alpha_{z}^{0} / \alpha_{\perp}^{0} \approx 6\right)$, the effectiveness of the VV corrections for the two directions is no surprise. As a function of temperature, we observe that the VV-corrected magnetizations in both geometries show increasing discrepancies at the same field as $T$ is increased to $70 \mathrm{~K}$ (Fig. 7), even though 
the nominal thermal cutoff imposed by the next CEF level is $\Delta_{10} / k_{B} \approx 160 \mathrm{~K}$.

Thus our experiments and analysis demonstrate two key results. The first is that all of the nontrivial magnetic properties of a material are captured by a single concept, the VV coefficients. The second is that the contrast between conventional and nontrivial magnetic properties can be found in a single material due to anisotropic VV coefficients that differ strongly between the field geometries $H \| a b$ and $H \| c$. We have shown that the origin of the VV coefficients lies in the large matrix elements governing the mixing and consequent repulsion of adjacent CEF levels. Although the ground-state VV coefficients, $\alpha_{\perp}^{0}$ and $\alpha_{z}^{0}$, are zero-field quantities whose effects can be found in non-CW behavior of the susceptibility, their strongest impact emerges as $H$ increases (Fig. 6). Our analysis also demonstrates that the success or failure of the popular pseudospin- $1 / 2$ approximation to the magnetic properties of a Kramers-doublet system depends directly on the magnitude of the VV coefficients.

Our conclusions rely completely on determining all of the CEF parameters with high accuracy, which allows a detailed characterization of all the magnetic properties throughout the $(H, \theta, T)$ parameter space within a single and self-consistent model. The simplest indication for a significant contribution from the VV coefficients is a deviation of the susceptibility from a CW form, which is visible most clearly as a downward curvature in $\chi^{-1}(T)$ as $T \rightarrow 0$; this type of behavior is obvious in $\chi_{c}^{-1}(T)$ in Fig. 5, but not in $\chi_{a b}^{-1}(T)$. As noted above, a more detailed characterization of the VV coefficients requires experiments under significant applied fields. Quantitatively, the origin of the $\mathrm{VV}$ coefficients in second-order perturbation theory [Eq. (7)] gives them a systematic dependence on $\Delta_{n 0}^{-1}$, as well as on the expectation values of $\hat{J}_{x(z)}$. Although the latter terms are primarily responsible for the strong directional anisotropy in the Yb-based delafossites whose reported $\Delta_{10}$ values and extracted VV coefficients are collected in Table I, the related material $\mathrm{YbMgGaO}_{4}$ presents an example where the large value of $\Delta_{10}$ suppresses the $\mathrm{VV}$ coefficients, leading to a predominantly $\mathrm{CW}$-type behavior of $\chi^{-1}(T)$ [34].

We stress again that our full microscopic model is directly applicable to the computation of all aspects of the magnetic response of a material. Here we have illustrated the situation for our own magnetic susceptibility, magnetization, and RTM data, and we await its extension to describe magnetic specific-heat, torque, ESR, and magnetocalorimetric measurements. We have also distilled the properties of the full model to the physically relevant VV coefficients, which can be understood as governing the nonlinear field dependence of the CEF energy levels (Fig. 6) and thus affect all of the magnetic properties. This linking function allows the use of our analysis to resolve a number of contradictions that have emerged in recent studies of delafossites by different techniques.

One example is the report from single-crystal INS measurements on $\mathrm{CsYbSe}_{2}$ of no CEF levels below $20 \mathrm{meV}$ [43], in direct contradiction to the present conclusions (Fig. 6). We have calculated the scattering cross-section for the $0 \rightarrow 1$ transition by using the diagonalized CEF matrix in order to compare the intensity obtained in the crystalline geometry of the measurement with that expected for a polycrystalline sample. Indeed we find the former to be smaller than the latter by four orders of magnitude, indicating that the initial conclusion was the consequence of an unfortunate choice of geometry, and subsequent reports suggest that a CEF level has been found around $15 \mathrm{meV}$ [54]. We note also that the two sets of Stevens operator coefficients proposed [21] for $\mathrm{NaYbO}_{2}$ based on INS measurements of the CEF spectrum have widely divergent VV coefficients, whereas similar fits to the INS spectra of $\mathrm{NaYbSe}_{2}$ show much more agreement when refined with complementary Raman scattering data [25].

\section{B. Magnetic interaction parameters and triangular-lattice spin models}

We turn our discussion from the Stevens operators determining the CEF levels to the magnetic interaction parameters that govern the low-energy physics, and hence the extent to which the Kramers-doublet system can be used as an effective realization of any of the paradigm $S=1 / 2$ models in quantum magnetism. As Sec. V A made clear, an adequate understanding of the CEF energies and their evolution in an applied field is a prerequisite in the search for phenomena including field-induced phase transitions and candidate QSL phases [1]. In particular, narrowly spaced CEF levels that undergo significant mutual repulsion at a specific field scale offer a complex and correlated energy landscape that could accommodate unconventional spin states.

Focusing on the triangular geometry, the nearest-neighbor triangular-lattice Heisenberg model is the original [55] and still one of the deepest problems in frustrated quantum magnetism [56,57]. Although the ground state of this model has a modest amount of magnetic order, triangular lattices have attracted extensive interest from a number of angles over the decades. Not only does this geometry have multiple materials realizations, but each generation of materials has opened a new dimension in research [56]. Triangular organic compounds drove a discussion of proximity to the Mott transition [5-8], $\mathrm{Cs}_{2} \mathrm{CuCl}_{4}$ and $\mathrm{Cs}_{2} \mathrm{CuBr}_{4}$ [58-62] drove studies of the spatially anisotropic $\left(J-J^{\prime}\right)$ triangular-lattice models [57,63], and the cobaltates $\mathrm{Ba}_{3} \mathrm{CoNb}_{2} \mathrm{O}_{9}$ [64,65], $\mathrm{Ba}_{3} \mathrm{CoSb}_{2} \mathrm{O}_{9}$ [66-68], and $\mathrm{Ba}_{8} \mathrm{CoNb}_{6} \mathrm{O}_{24}$ [69] spurred the consideration of spin-anisotropic triangular lattices with $\mathrm{XXZ}$ symmetry. In recent years, Yb-based triangular-lattice materials have sparked very strong interest in further spin anisotropies, in the form of $J^{++}$and $J^{+z}$ terms [38,48,70], all of which widen considerably the scope for finding QSL states. On the theoretical side, it has been shown that secondneighbor Heisenberg interactions also drive a QSL state, whose gapped or gapless nature remains undetermined at present [71-75]. In the nearest-neighbor model, the systematic treatment of parton-based formulations has led to qualitative advances in calculating the dynamical excitation spectrum by both Schwinger-boson [76] and pseudofermion methods [73,77]. Long-standing questions about the thermodynamic properties may soon be answered by DMRG methods [78], despite the constraints of working on a rather narrow cylinder, and by tensor-network methods [79] despite the challenge posed by the high connectivity of the triangular lattice. 
In Sec. II, we restricted our considerations to a triangularlattice model of XXZ form [Eq. (2)], meaning that we allowed only a minimal spin anisotropy of Ising or XY form. Working at the mean-field level [Eq. (5)], in Sec. III B, we obtained the results $\mathcal{J}_{\perp}=0.54 \pm 0.01 \mathrm{~K}$ and $\mathcal{J}_{z}=0.61 \pm 0.01 \mathrm{~K}$ for the magnetic interactions in the system of $J=7 / 2 \mathrm{Yb}^{3+}$ ions. As shown in Appendix $\mathrm{C}$, the interaction terms of the corresponding effective pseudospin- $1 / 2$ model are $\mathcal{J}_{\perp}^{\prime}=$ $5.12 \mathrm{~K}(\simeq 0.44 \mathrm{meV})$ and $\mathcal{J}_{z}^{\prime}=0.84 \mathrm{~K}(\simeq 0.07 \mathrm{meV})$, meaning that $\mathrm{CsYbSe}_{2}$ is very strongly in the $\mathrm{XY}$ limit $\left(\mathcal{J}_{z}^{\prime} / \mathcal{J}_{\perp}^{\prime} \simeq\right.$ 0.16). Although this contrasts with most assumptions made at present, we note that XY character has also been deduced for each of the $\mathrm{NaYb} X_{2}$ materials by using a pseudospin-1/2 treatment [30]. We expect further investigations of $\mathrm{CsYbSe}_{2}$ to confirm this result. As the most straightforward indicator of XY or Ising physics, we suggest that the width in field of the regime over which the $1 / 3$ plateau (i.e. the state of up-updown spin order) in the magnetization is stabilized constitutes a quantity rather sensitive to the ratio $\mathcal{J}_{z}^{\prime} / \mathcal{J}_{\perp}^{\prime}[47,80]$. This plateau is evident in the data of Ref. [43], although a lower temperature would assist a quantitative analysis. However, the relevant regime of parameter space is yet to be investigated theoretically for a field applied in the $a b$ plane of the system. Finally, while we cannot exclude terms of $J^{++}$and $J^{+z}$ type from the spin Hamiltonian, the accuracy of our fit indicates that the effect of any missing terms is extremely small in $\mathrm{CsYbSe}_{2}$; either they are genuinely small or they are relevant only at the lowest temperatures where a treatment beyond the present mean-field level would be required.

On the materials side, it is also fair to say that the continuing lack of experimental signatures for QSL ground states can be blamed on two primary issues, namely the scarcity of candidate materials and the paucity of measurable physical quantities offering unambiguous signatures or predictors for QSL properties (such as fractional quantum numbers and nonlocal entanglement). To address the first of these, our analysis provides a definitive guide to the field-induced physics of highly spin-anisotropic systems such as the Yb-delafossites, and hence to the regions of parameter space where competing energy scales establish an environment conducive to the occurrence of exotic spin states. Although we cannot solve the second issue, we can provide a comprehensive understanding of the single-ion energy spectrum that identifies the extent to which a given material replicates a target pseudospin-1/2 model, thereby streamlining the experimental search for QSL fingerprints by available experimental methods.

\section{SUMMARY}

We have investigated the anisotropic magnetic response of an insulating $4 f$ electronic system by measuring two key thermodynamic quantities, the magnetic susceptibility in the low-field limit and the magnetotropic coefficients over very wide field and temperature ranges (up to $\mu_{0} H=60 \mathrm{~T}$ and $T=70 \mathrm{~K}$ ). We have shown that the anisotropies in both quantities can be formulated within a set of anisotropic van Vleck (VV) coefficients, which arise as the second-order perturbative corrections of the Zeeman interaction to the zero-field crystal electric field (CEF) spectrum. This leads to the essential finding that the VV coefficients constitute independent physical quantities that describe the crucial magnetic properties of $4 f$ spin systems across the full range of applied fields and extant anisotropies. A proper account of the groundstate VV coefficients is indispensable for an accurate and unambiguous determination of the microscopic parameters governing the CEF Hamiltonian, a process for which otherwise few routes are known to date. The VV coefficients fulfill the vital function of unifying the low-field, low-temperature magnetic susceptibility with the high-field magnetotropic coefficients and CEF levels, and in this sense their role as stand-alone physical quantities allowing a full interpretation of magnetic anisotropies has not been appreciated before.

Our experimental results highlight the value of the resonant torsion magnetometry (RTM) method, which is accurate and profoundly powerful in terms of the parameter ranges it accesses. The magnetotropic coefficients we extract over these broad field and temperature ranges play the key role in obtaining a unique set of Stevens operators describing the microscopic CEF Hamiltonian with unprecedent fidelity. We reiterate that a fitting analysis must provide complete consistency from zero to high field and at all relevant temperatures, and our fits meet this challenge. With the full CEF spectrum in hand, we can examine the validity of different and popular approximations that have been applied to many materials. Specifically, we identify the limits of a CW fit to the temperature dependence of the magnetic susceptibility and the boundaries of the effective pseudospin-1/2 description for systems with a ground-state Kramers doublet.

We have developed and applied our analysis for the material $\mathrm{CsYbSe}_{2}$, which is a member of a family of $\mathrm{Yb}$ delafossites displaying triangular-lattice geometry. Because the CEF levels of the $\mathrm{Yb}^{3+}$ ion are four Kramers doublets, these compounds are leading candidates in the search for quantum spin-liquid (QSL) behavior, and indeed the full CEF spectrum we obtain up to high fields (Fig. 6) reveals an intricate and anisotropic energy landscape amenable to unconventional magnetism. This spectrum allows one to construct a maximally informed pseudospin-1/2 model for the lowenergy physics of the system, and within a minimal XXZ spin Hamiltonian we conclude that $\mathrm{CsYbSe}_{2}$ is a strongly $\mathrm{XY}$ triangular antiferromagnet. While we await further experimental confirmation of this result, we note again that our analysis is applicable to a wide range of $4 f$ materials with complex $\mathrm{CEF}$ spectra and especially with ground-state doublets allowing an effective spin-1/2 description, which should expand significantly the scope of the search for QSL phases.

\section{ACKNOWLEDGMENTS}

We thank M. Mourigal, S. Nikitin, and K. Ross for helpful discussions. We are grateful to M. Chan and A. Shekhter for technical assistance with our pulsed-field measurements. Experimental work at the University of Colorado Boulder was supported by Award No. DE-SC0021377 of the U.S. Department of Energy (DOE), Basic Energy Sciences (BES), Materials Sciences and Engineering Division (MSE). Theoretical work at the University of Colorado Boulder was supported by Award No. DE-SC0014415 of the U.S. DOE, BES, MSE. Work at Oak Ridge National Laboratory was supported by the U.S. DOE, BES, MSE. A portion of this work was performed 
at the National High Magnetic Field Laboratory, in the Pulsed Field Facility at Los Alamos National Laboratory, which is supported by National Science Foundation Cooperative Agreement No. DMR-1644779, the State of Florida, and the U.S. DOE. The publication of this article was funded partially by the University of Colorado Boulder Libraries Open Access Fund.

\section{APPENDIX A: EXACT FORM OF MAGNETIC SUSCEPTIBILITY}

From the $N$-particle partition function calculated with Eq. (5), we derive analytical formulas for the low-field magnetic susceptibilities of our model using the thermodynamic relation

$$
\chi_{a b(c)}(T)=-\left.\frac{1}{\mu_{0}} \frac{\partial^{2} F}{\partial H^{2}}\right|_{H_{a b, c} \rightarrow 0}=\frac{N \mu_{0} \mu_{B}^{2} g_{J}^{2}}{k_{B}}\left[\frac{T \sum_{n=0}^{3} e^{-\beta E_{n}}}{\sum_{n=0}^{3}\left(\left|\left\langle n_{+}\left|\hat{J}_{x(z)}\right| n_{-(+)}\right\rangle\right|^{2}-\alpha_{\perp(z)}^{n} \frac{2 k_{B} T}{\mu_{0}^{2} \mu_{B}^{2} g_{J}^{2}}\right) e^{-\beta E_{n}}}+\Theta_{\perp(z)}^{\mathrm{CW}}\right]^{-1}
$$

where $\beta=1 / k_{B} T, \Theta_{\perp(z)}^{\mathrm{CW}}=q \mathcal{J}_{\perp(z)} / k_{B}$ as in Eq. (8), and $\alpha_{i}^{n}$ are the VV coefficients defined in Eq. (7). The form of Eq. (A1) is exact within the mean-field approximation, and by considering only $n=0$ (the ground-state doublet) it reduces to Eq. (8).

\section{APPENDIX B: COMPARISON TO THE CURIE-WEISS MAGNETIC SUSCEPTIBILITY OF A SPIN-1/2 SYSTEM}

To illustrate how the VV coefficients alter even the low$T$ limit of the full susceptibility expression, where only the lowest Kramers doublet is relevant, we repeat Eq. (8) in the form

$\chi_{a b(c)}^{-1}=\frac{k_{B}}{N \mu_{0} \mu_{B}^{2} g_{J}^{2}}\left[\frac{T}{\left(\frac{g_{\perp(z)}}{2 g_{J}}\right)^{2}-\frac{2 k_{B} \alpha_{\perp(z)}^{0}}{\mu_{0}^{2} \mu_{B}^{2} g_{J}^{2}} T}+\Theta_{\perp(z)}^{\mathrm{CW}}\right]$.

Only when the VV term is negligible does this expression reduce to the familiar $\mathrm{CW}$ form,

$$
\chi_{a b(c)}^{\mathrm{CW}}=\frac{N \mu_{0} \mu_{B}^{2} g_{\perp(z)}^{2}}{4 k_{B}}\left(T+\widetilde{\Theta}_{\perp(z)}^{\mathrm{CW}}\right)^{-1}
$$

with a $\mathrm{CW}$ temperature for the effective $S=1 / 2$ model given by $q \mathcal{J}^{\prime} \perp(z) / 4 k_{B}$, where the parameters $\mathcal{J}^{\prime}{ }_{\perp}$ and $\mathcal{J}^{\prime}{ }_{z}$ are defined in Appendix $\mathrm{C}$ below.

\section{APPENDIX C: EFFECTIVE SPIN-1/2 PROJECTION}

At the lowest temperatures, where the physics is dominated by the properties of the lowest Kramers doublet, $\left\{\left|0_{ \pm}\right\rangle\right\}$, it is helpful to formulate a pseudospin-1/2 model in terms of effective spin- $1 / 2$ operators with the action

$$
\begin{aligned}
& \hat{S}_{x}=\frac{1}{2}\left(\left|0_{+}\right\rangle\left\langle 0_{-}|+| 0_{-}\right\rangle\left\langle 0_{+}\right|\right), \\
& \hat{S}_{z}=\frac{1}{2}\left(\left|0_{+}\right\rangle\left\langle 0_{+}|-| 0_{-}\right\rangle\left\langle 0_{-}\right|\right) .
\end{aligned}
$$

The equivalent pseudospin-1/2 model for a Hamiltonian $\hat{\mathcal{H}}$ is obtained formally by projecting onto the lowest Kramersdoublet subspace using the projection operator

$$
\hat{P}_{0} \equiv\left|0_{+}\right\rangle\left\langle 0_{+}|+| 0_{-}\right\rangle\left\langle 0_{-}\right| \text {. }
$$

For the full $J=7 / 2$ Hamiltonian in the form

$$
\hat{\mathcal{H}}=\sum_{\langle i, j\rangle}\left(\mathcal{J}_{\perp} \hat{J}_{i, x} \hat{J}_{j, x}+\mathcal{J}_{z} \hat{J}_{i, z} \hat{J}_{j, z}\right)+\mu_{0} \mu_{B} g_{J} \mathbf{H} \cdot \sum_{i} \hat{\mathbf{J}}_{i},
$$

the equivalent pseudospin-1/2 model is

$$
\begin{gathered}
\hat{\mathcal{H}}_{\mathrm{PS}}=\hat{P}_{0} \hat{\mathcal{H}} \hat{P}_{0}, \\
=\sum_{\langle i, j\rangle}\left(\mathcal{J}_{\perp}^{\prime} \hat{S}_{i, x} \hat{S}_{j, x}+\mathcal{J}_{z}^{\prime} \hat{S}_{i, z} \hat{S}_{j, z}\right) \\
+\mu_{0} \mu_{B} \sum_{i}\left(g_{\perp} H_{\perp} \hat{S}_{i, x}+g_{z} H_{z} \hat{S}_{i, z}\right),
\end{gathered}
$$

from which it follows that the equivalent $g$ factors and exchange constants of the pseudospin- $1 / 2$ model are related to $J=7 / 2$ operator matrix elements and exchange constants by the expressions

$$
\begin{gathered}
g_{\perp(z)}=2 g_{J}\left|\left\langle 0_{ \pm}\left|\hat{J}_{x(z)}\right| 0_{\mp( \pm)}\right\rangle\right|, \\
\mathcal{J}_{\perp(z)}^{\prime}=\left(\frac{g_{\perp(z)}}{g_{J}}\right)^{2} \mathcal{J}_{\perp(z)} .
\end{gathered}
$$

Applying these relations using $g_{J}=8 / 7$ gives the $g$ factors $g_{\perp}=3.77$ and $g_{z}=1.76$ shown in the second line of Table II, and using the results $\mathcal{J}_{\perp}=0.54 \pm 0.01 \mathrm{~K}$ and $\mathcal{J}_{z}=0.61 \pm$ $0.01 \mathrm{~K}$ obtained in Sec. III B for the $J=7 / 2$ system gives the values $\mathcal{J}_{\perp}^{\prime}=5.12 \mathrm{~K}(\simeq 0.44 \mathrm{meV})$ and $\mathcal{J}_{z}^{\prime}=0.84 \mathrm{~K}(\simeq$ $0.07 \mathrm{meV}$ ) for the interaction parameters of the pseudospin$1 / 2$ model.

\section{APPENDIX D: MAGNETOTROPIC COEFFICIENTS}

In an anisotropic magnetic material, a magnetic-fieldangle-dependent contribution to the Helmholtz free energy,

$$
F(T, H, \vartheta)=-\frac{1}{\beta} \ln \operatorname{Tr}\left[e^{-\beta \hat{\mathcal{H}}(H, \vartheta)}\right],
$$

can be modelled using the explicit field dependence of the microscopic spin Hamiltonian, $\hat{\mathcal{H}}(H, \vartheta)$. In Sec. II, we model this at the mean-field level, using $\hat{\mathcal{H}}_{\mathrm{MF}}^{\mathrm{sg}}[$ Eq. (5)]. RTM is a direct probe of the curvature of the magnetic free energy with respect to changes in the angle at which the field is applied, 
and this curvature is defined as the magnetotropic coefficient,

$$
k(H, \vartheta)=\frac{\partial^{2} F}{\partial \vartheta^{2}}=-\frac{\partial \tau(H, \vartheta)}{\partial \vartheta},
$$

where $\tau(H, \vartheta)=\mathbf{M} \times \mathbf{H}$ is the magnetic torque and $\vartheta$ is the angular direction of $H$ measured in the plane of vibration of the sample. For the system of a piezoresistive cantilever with a tip-mounted sample, the angle-dependent magnetotropic coefficient at finite field produces the proportional frequency shift specified in Eq. (9) [45,46]. For the cases considered here, we define the total vector magnetization in a finite field with components $H_{\perp}=H \sin \theta$ and $H_{z}=H \cos \theta$ in terms of its component magnetizations as

$$
\mathbf{M}(\mathbf{H})=M_{a b}\left(H_{\perp}, H_{z}\right) \hat{\mathbf{x}}+M_{c}\left(H_{\perp}, H_{z}\right) \hat{\mathbf{z}} .
$$

The torque as function of angle then takes the explicit form

$$
\tau(\theta)=\mathbf{M} \times \mathbf{H}=M_{a b} H \cos \theta-M_{c} H \sin \theta .
$$

In addition to the conventional susceptibilities,

$$
\begin{aligned}
\chi_{a b} & =\lim _{h \rightarrow 0} \frac{M_{a b}\left(H_{\perp}=h, H_{z}=0\right)}{h}, \\
\chi_{c} & =\lim _{h \rightarrow 0} \frac{M_{c}(0, h)}{h},
\end{aligned}
$$

one may define the transverse susceptibilities at finite field, for a magnetization induced by a small field $h$ applied perpendicular to an arbitrarily large (but finite) field $H$, as

$$
\begin{aligned}
\chi_{a b}^{T}(H)= & \lim _{h \rightarrow 0} \frac{M_{a b}\left(H_{\perp}=h, H_{z}=H\right)-M_{a b}(0, H)}{h}, \\
& \chi_{c}^{T}(H)=\lim _{h \rightarrow 0} \frac{M_{c}(H, h)-M_{c}(H, 0)}{h} .
\end{aligned}
$$

With these definitions we express the magnetotropic coefficients at the high-symmetry angles as $k_{a b}(H)=k(H, \theta=$ $\pi / 2)$ and $k_{c}(H)=k(H, \theta=0)$, with

$$
\begin{gathered}
k_{a b}(H)=H M_{a b}(H, 0)-H^{2} \chi_{c}^{T}(H), \\
k_{c}(H)=H M_{c}(0, H)-H^{2} \chi_{a b}^{T}(H) .
\end{gathered}
$$

In the low-field limit, the transverse susceptibilities are in fact identical to their zero-field values, so that $k_{a b}=-k_{c}=\left(\chi_{a b}\right.$ $\left.\chi_{c}\right) H^{2}$. The low-field expressions are no longer valid when the magnetization ceases to be $H$-linear, necessitating a more detailed description in terms of Eqs. (D1) and (D2). From the expressions at low and high fields, one may interpret the magnetotropic coefficient at a given applied-field angle, $\theta$, as the energy difference between the real magnetostatic potential energy at that angle and the "naive" energy cost of magnetizing the sample in a completely perpendicular direction based on a linear extrapolation of the transverse susceptibility.
[1] L. Savary and L. Balents, Quantum spin liquids: A review, Rep. Prog. Phys. 80, 016502 (2016).

[2] J. S. Helton, K. Matan, M. P. Shores, E. A. Nytko, B. M. Bartlett, Y. Yoshida, Y. Takano, A. Suslov, Y. Qiu, J.-H. Chung, D. G. Nocera, and Y. S. Lee, Spin Dynamics of the Spin-1/2 Kagome Lattice Antiferromagnet $\mathrm{ZnCu}_{3}(\mathrm{OH})_{6} \mathrm{Cl}_{2}$, Phys. Rev. Lett. 98, 107204 (2007).

[3] J. S. Gardner, M. J. P. Gingras, and J. E. Greedan, Magnetic pyrochlore oxides, Rev. Mod. Phys. 82, 53 (2010).

[4] J. G. Rau and M. J. Gingras, Frustrated quantum rare-earth pyrochlores, Annu. Rev. Condens. Matter Phys. 10, 357 (2019).

[5] Y. Shimizu, K. Miyagawa, K. Kanoda, M. Maesato, and G. Saito, Spin Liquid State in an Organic Mott Insulator with a Triangular Lattice, Phys. Rev. Lett. 91, 107001 (2003).

[6] S. Yamashita, T. Yamamoto, Y. Nakazawa, M. Tamura, and R. Kato, Gapless spin liquid of an organic triangular compound evidenced by thermodynamic measurements, Nat. Commun. 2 , 275 (2011)

[7] T. Itou, A. Oyamada, S. Maegawa, M. Tamura, and R. Kato, Quantum spin liquid in the spin-1/2 triangular antiferromagnet $\mathrm{EtMe}_{3} \mathrm{Sb}\left[\mathrm{Pd}(\mathrm{dmit})_{2}\right]_{2}$, Phys. Rev. B 77, 104413 (2008).

[8] T. Itou, A. Oyamada, S. Maegawa, and R. Kato, Instability of a quantum spin liquid in an organic triangular-lattice antiferromagnet, Nat. Phys. 6, 673 (2010).

[9] K. Matsuhira, M. Wakeshima, Y. Hinatsu, and S. Takagi, Metalinsulator transitions in pyrochlore oxides $\mathrm{Ln}_{2} \mathrm{Ir}_{2} \mathrm{O}_{7}$, J. Phys. Soc. Jpn. 80, 094701 (2011).

[10] G. Chen and M. Hermele, Magnetic orders and topological phases from $f$ - $d$ exchange in pyrochlore iridates, Phys. Rev. B 86, 235129 (2012).
[11] G. Jackeli and G. Khaliullin, Mott Insulators in the Strong Spin-Orbit Coupling Limit: From Heisenberg to a Quantum Compass and Kitaev Models, Phys. Rev. Lett. 102, 017205 (2009).

[12] S. K. Choi, R. Coldea, A. N. Kolmogorov, T. Lancaster, I. I. Mazin, S. J. Blundell, P. G. Radaelli, Y. Singh, P. Gegenwart, K. R. Choi, S.-W. Cheong, P. J. Baker, C. Stock, and J. Taylor, Spin Waves and Revised Crystal Structure of Honeycomb Iridate $\mathrm{Na}_{2} \mathrm{IrO}_{3}$, Phys. Rev. Lett. 108, 127204 (2012).

[13] Y. Singh, S. Manni, J. Reuther, T. Berlijn, R. Thomale, W. $\mathrm{Ku}, \mathrm{S}$. Trebst, and P. Gegenwart, Relevance of the HeisenbergKitaev Model for the Honeycomb Lattice Iridates $A_{2} \mathrm{IrO}_{3}$, Phys. Rev. Lett. 108, 127203 (2012).

[14] K. W. Plumb, J. P. Clancy, L. J. Sandilands, V. V. Shankar, Y. F. $\mathrm{Hu}, \mathrm{K}$. S. Burch, H.-Y. Kee, and Y.-J. Kim, $\alpha-\mathrm{RuCl}_{3}$ : A spinorbit assisted Mott insulator on a honeycomb lattice, Phys. Rev. B 90, 041112(R) (2014).

[15] S. H. Chun, J.-W. Kim, J. Kim, H. Zheng, C. C. Stoumpos, C. D. Malliakas, J. F. Mitchell, K. Mehlawat, Y. Singh, Y. Choi, T. Gog, A. Al-Zein, M. Sala, M. Krisch, J. Chaloupka, G. Jackeli, G. Khaliullin, and B. J. Kim, Direct evidence for dominant bond-directional interactions in a honeycomb lattice iridate $\mathrm{Na}_{2} \mathrm{IrO}_{3}$, Nat. Phys. 11, 462 (2015).

[16] B. Gao, T. Chen, D. W. Tam, C.-L. Huang, K. Sasmal, D. T. Adroja, F. Ye, H. Cao, G. Sala, M. B. Stone, C. Baines, J. A. T. Verezhak, H. Hu, J.-H. Chung, X. Xu, S.-W. Cheong, M. Nallaiyan, S. Spagna, M. B. Maple, A. H. Nevidomskyy et al., Experimental signatures of a three-dimensional quantum spin liquid in effective spin-1/2 $\mathrm{Ce}_{2} \mathrm{Zr}_{2} \mathrm{O}_{7}$ pyrochlore, Nat. Phys. 15, 1052 (2018). 
[17] C. Mauws, A. M. Hallas, G. Sala, A. A. Aczel, P. M. Sarte, J. Gaudet, D. Ziat, J. A. Quilliam, J. A. Lussier, M. Bieringer, H. D. Zhou, A. Wildes, M. B. Stone, D. Abernathy, G. M. Luke, B. D. Gaulin, and C. R. Wiebe, Dipolar-octupolar Ising antiferromagnetism in $\mathrm{Sm}_{2} \mathrm{Ti}_{2} \mathrm{O}_{7}$ : A moment fragmentation candidate, Phys. Rev. B 98, 100401(R) (2018).

[18] A. Scheie, V. O. Garlea, L. D. Sanjeewa, J. Xing, and A. S. Sefat, Crystal-field Hamiltonian and anisotropy in $\mathrm{KErSe}_{2}$ and $\mathrm{CsErSe}_{2}$, Phys. Rev. B 101, 144432 (2020).

[19] A. Scheie, PyCrystalField: Software for calculation, analysis, and fitting of crystal electric field Hamiltonians, J. Appl. Crystallogr. 54, 356 (2021).

[20] R. Sibille, E. Lhotel, V. Pomjakushin, C. Baines, T. Fennell, and M. Kenzelmann, Candidate Quantum Spin Liquid in the $\mathrm{Ce}^{3+}$ Pyrochlore Stannate $\mathrm{Ce}_{2} \mathrm{Sn}_{2} \mathrm{O}_{7}$, Phys. Rev. Lett. 115, 097202 (2015).

[21] M. M. Bordelon, E. Kenney, C. Liu, T. Hogan, L. Posthuma, M. Kavand, Y. Lyu, M. Sherwin, N. P. Butch, C. Brown, M. J. Graf, L. Balents, and S. D. Wilson, Field-tunable quantum disordered ground state in the triangular-lattice antiferromagnet $\mathrm{NaYbO}_{2}$, Nat. Phys. 15, 1058 (2019).

[22] P.-L. Dai, G. Zhang, Y. Xie, C. Duan, Y. Gao, Z. Zhu, E. Feng, Z. Tao, C.-L. Huang, H. Cao, A. Podlesnyak, G. E. Granroth, M. S. Everett, J. C. Neuefeind, D. Voneshen, S. Wang, G. Tan, E. Morosan, X. Wang, H.-Q. Lin et al., Spinon Fermi Surface Spin Liquid in a Triangular Lattice Antiferromagnet $\mathrm{NaYbSe}_{2}$, Phys. Rev. X 11, 021044 (2021).

[23] M. Baenitz, P. Schlender, J. Sichelschmidt, Y. A. Onykiienko, Z. Zangeneh, K. M. Ranjith, R. Sarkar, L. Hozoi, H. C. Walker, J.-C. Orain, H. Yasuoka, J. van den Brink, H. H. Klauss, D. S. Inosov, and T. Doert, $\mathrm{NaYbS}_{2}$ : A planar spin- $\frac{1}{2}$ triangular-lattice magnet and putative spin liquid, Phys. Rev. B 98, 220409(R) (2018).

[24] K. M. Ranjith, D. Dmytriieva, S. Khim, J. Sichelschmidt, S. Luther, D. Ehlers, H. Yasuoka, J. Wosnitza, A. A. Tsirlin, H. Kühne, and M. Baenitz, Field-induced instability of the quantum spin liquid ground state in the $J_{\text {eff }}=\frac{1}{2}$ triangular-lattice compound $\mathrm{NaYbO}_{2}$, Phys. Rev. B 99, 180401(R) (2019).

[25] K. M. Ranjith, S. Luther, T. Reimann, B. Schmidt, P. Schlender, J. Sichelschmidt, H. Yasuoka, A. M. Strydom, Y. Skourski, J. Wosnitza, H. Kühne, T. Doert, and M. Baenitz, Anisotropic field-induced ordering in the triangular-lattice quantum spin liquid $\mathrm{NaYbSe}_{2}$, Phys. Rev. B 100, 224417 (2019).

[26] R. Sarkar, P. Schlender, V. Grinenko, E. Haeussler, P. J. Baker, T. Doert, and H.-H. Klauss, Quantum spin liquid ground state in the disorder free triangular lattice $\mathrm{NaYbS}_{2}$, Phys. Rev. B 100, 241116(R) (2019).

[27] J. Sichelschmidt, B. Schmidt, P. Schlender, S. Khim, T. Doert, and M. Baenitz, Effective spin-1/2 moments on a $\mathrm{Yb}^{3+}$ triangular lattice: An ESR study, JPS Conf. Proc. 30, 011096 (2020).

[28] M. M. Bordelon, C. Liu, L. Posthuma, P. M. Sarte, N. P. Butch, D. M. Pajerowski, A. Banerjee, L. Balents, and S. D. Wilson, Spin excitations in the frustrated triangular lattice antiferromagnet $\mathrm{NaYbO}_{2}$, Phys. Rev. B 101, 224427 (2020).

[29] Z. Zhang, X. Ma, J. Li, G. Wang, D. T. Adroja, T. P. Perring, W. Liu, F. Jin, J. Ji, Y. Wang, Y. Kamiya, X. Wang, J. Ma, and Q. Zhang, Crystalline electric field excitations in the quantum spin liquid candidate $\mathrm{NaYbSe}_{2}$, Phys. Rev. B 103, 035144 (2021).
[30] B. Schmidt, J. Sichelschmidt, K. M. Ranjith, T. Doert, and M. Baenitz, $\mathrm{Yb}$ delafossites: Unique exchange frustration of $4 f$ spin-1/2 moments on a perfect triangular lattice, Phys. Rev. B 103, 214445 (2021).

[31] Y. Li, G. Chen, W. Tong, L. Pi, J. Liu, Z. Yang, X. Wang, and Q. Zhang, Rare-Earth Triangular Lattice Spin Liquid: A SingleCrystal Study of $\mathrm{YbMgGaO}_{4}$, Phys. Rev. Lett. 115, 167203 (2015).

[32] Y. Shen, Y.-D. Li, H. Wo, Y. Li, S. Shen, B. Pan, Q. Wang, H. C. Walker, P. Steffens, M. Boehm, Y. Hao, D. L. Quintero-Castro, L. W. Harriger, M. D. Frontzek, L. Hao, S. Meng, Q. Zhang, G. Chen, and J. Zhao, Evidence for a spinon Fermi surface in a triangular-lattice quantum-spin-liquid candidate, Nature (London) 540, 559 (2016).

[33] Y.-D. Li, X. Wang, and G. Chen, Anisotropic spin model of strong spin-orbit-coupled triangular antiferromagnets, Phys. Rev. B 94, 035107 (2016).

[34] J. A. M. Paddison, M. Daum, Z. Dun, G. Ehlers, Y. Liu, M. B. Stone, H. Zhou, and M. Mourigal, Continuous excitations of the triangular-lattice quantum spin liquid $\mathrm{YbMgGaO}_{4}$, Nat. Phys. 13, 117 (2017).

[35] Y.-D. Li, Y. Shen, Y. Li, J. Zhao, and G. Chen, Effect of spinorbit coupling on the effective-spin correlation in $\mathrm{YbMgGaO}_{4}$, Phys. Rev. B 97, 125105 (2018).

[36] Y. Shen, Y.-D. Li, H. C. Walker, P. Steffens, M. Boehm, X. Zhang, S. Shen, H. Wo, G. Chen, and J. Zhao, Fractionalized excitations in the partially magnetized spin liquid candidate $\mathrm{YbMgGaO}_{4}$, Nat. Commun. 9, 4138 (2018).

[37] Y. Li, D. Adroja, R. I. Bewley, D. Voneshen, A. A. Tsirlin, P. Gegenwart, and Q. Zhang, Crystalline Electric-Field Randomness in the Triangular Lattice Spin-Liquid $\mathrm{YbMgGaO}_{4}$, Phys. Rev. Lett. 118, 107202 (2017).

[38] Z. Zhu, P. A. Maksimov, S. R. White, and A. L. Chernyshev, Disorder-Induced Mimicry of a Spin Liquid in $\mathrm{YbMgGaO}_{4}$, Phys. Rev. Lett. 119, 157201 (2017).

[39] T. Xie, J. Xing, S. E. Nikitin, S. Nishimoto, M. Brando, P. Khanenko, J. Sichelschmidt, L. D. Sanjeewa, A. S. Sefat, and A. Podlesnyak, Field-induced spin excitations in the spin-1/2 triangular-lattice antiferromagnet $\mathrm{CsYbSe}_{2}$, arXiv:2106.12451.

[40] J. Banda, B. K. Rai, H. Rosner, E. Morosan, C. Geibel, and M. Brando, Crystalline electric field of $\mathrm{Ce}$ in trigonal symmetry: $\mathrm{CeIr}_{3} \mathrm{Ge}_{7}$ as a model case, Phys. Rev. B 98, 195120 (2018).

[41] B.-Q. Liu, P. Čermák, C. Franz, C. Pfleiderer, and A. Schneidewind, Lattice dynamics and coupled quadrupolephonon excitations in $\mathrm{CeAuAl}_{3}$, Phys. Rev. B 98, 174306 (2018).

[42] P. Čermák, A. Schneidewind, B. Liu, M. M. Koza, C. Franz, R. Schönmann, O. Sobolev, and C. Pfleiderer, Magnetoelastic hybrid excitations in $\mathrm{CeAuAl}_{3}$, Proc. Natl. Acad. Sci. 116, 6695 (2019).

[43] J. Xing, L. D. Sanjeewa, J. Kim, G. R. Stewart, A. Podlesnyak, and A. S. Sefat, Field-induced magnetic transition and spin fluctuations in the quantum spin-liquid candidate $\mathrm{CsYbSe}_{2}$, Phys. Rev. B 100, 220407(R) (2019).

[44] J. Xing, L. D. Sanjeewa, J. Kim, G. R. Stewart, M.-H. Du, F. A. Reboredo, R. Custelcean, and A. S. Sefat, Crystal synthesis and frustrated magnetism in triangular lattice $\mathrm{CsRESe}_{2}(\mathrm{RE}=\mathrm{La}-$ $\mathrm{Lu}$ ): Quantum spin liquid candidates $\mathrm{CsCeSe}_{2}$ and $\mathrm{CsYbSe}_{2}$, ACS Mater. Lett. 2, 71 (2020). 
[45] K. A. Modic, M. D. Bachmann, B. J. Ramshaw, F. Arnold, K. R. Shirer, A. Estry, J. B. Betts, N. J. Ghimire, E. D. Bauer, M. Schmidt, M. Baenitz, E. Svanidze, R. D. McDonald, A. Shekhter, and P. J. W. Moll, Resonant torsion magnetometry in anisotropic quantum materials, Nat. Commun. 9, 3975 (2018).

[46] K. A. Modic, R. D. McDonald, J. P. C. Ruff, M. D. Bachmann, Y. Lai, J. C. Palmstrom, D. Graf, M. K. Chan, F. F. Balakirev, J. B. Betts, G. S. Boebinger, M. Schmidt, M. J. Lawler, D. A. Sokolov, P. J. W. Moll, B. J. Ramshaw, and A. Shekhter, Scaleinvariant magnetic anisotropy in $\mathrm{RuCl}_{3}$ at high magnetic fields, Nat. Phys. 17, 240 (2020).

[47] D. Yamamoto, G. Marmorini, and I. Danshita, Quantum Phase Diagram of the Triangular-Lattice $X X Z$ Model in a Magnetic Field, Phys. Rev. Lett. 112, 127203 (2014).

[48] P. A. Maksimov, Z. Zhu, S. R. White, and A. L. Chernyshev, Anisotropic-Exchange Magnets on a Triangular Lattice: Spin Waves, Accidental Degeneracies, and Dual Spin Liquids, Phys. Rev. X 9, 021017 (2019).

[49] J. Gaudet, E. M. Smith, J. Dudemaine, J. Beare, C. R. C. Buhariwalla, N. P. Butch, M. B. Stone, A. I. Kolesnikov, G. Xu, D. R. Yahne, K. A. Ross, C. A. Marjerrison, J. D. Garrett, G. M. Luke, A. D. Bianchi, and B. D. Gaulin, Quantum Spin Ice Dynamics in the Dipole-Octupole Pyrochlore Magnet $\mathrm{Ce}_{2} \mathrm{Zr}_{2} \mathrm{O}_{7}$, Phys. Rev. Lett. 122, 187201 (2019).

[50] M. Doerr, M. Rotter, and A. Lindbaum, Magnetostriction in rare-earth based antiferromagnets, Adv. Phys. 54, 1 (2005).

[51] Y.-P. Huang, G. Chen, and M. Hermele, Quantum Spin Ices and Topological Phases from Dipolar-Octupolar Doublets on the Pyrochlore Lattice, Phys. Rev. Lett. 112, 167203 (2014).

[52] Y.-D. Li and G. Chen, Symmetry enriched U(1) topological orders for dipole-octupole doublets on a pyrochlore lattice, Phys. Rev. B 95, 041106(R) (2017).

[53] This can be understood by examining the eigenvalues of the threefold rotation operator, $\exp \left(i \frac{2 \pi J_{z}}{3}\right)$.

[54] S. Nikitin (private communication).

[55] P. W. Anderson, Resonating valence bonds: A new kind of insulator? Mat. Res. Bull. 8, 153 (1973).

[56] Y. Li, P. Gegenwart, and A. A. Tsirlin, Spin liquids in geometrically perfect triangular antiferromagnets, J. Phys.: Condens. Matter 32, 224004 (2020).

[57] O. Starykh, Unusual ordered phases of highly frustrated magnets: a review, Rep. Prog. Phys. 78, 052502 (2015).

[58] R. Coldea, D. A. Tennant, A. M. Tsvelik, and Z. Tylczynski, Experimental Realization of a 2D Fractional Quantum Spin Liquid, Phys. Rev. Lett. 86, 1335 (2001).

[59] R. Coldea, D. A. Tennant, K. Habicht, P. Smeibidl, C. Wolters, and Z. Tylczynski, Direct Measurement of the Spin Hamiltonian and Observation of Condensation of Magnons in the 2D Frustrated Quantum Magnet $\mathrm{Cs}_{2} \mathrm{CuCl}_{4}$, Phys. Rev. Lett. 88, 137203 (2002).

[60] R. Coldea, D. A. Tennant, and Z. Tylczynski, Extended scattering continua characteristic of spin fractionalization in the two-dimensional frustrated quantum magnet $\mathrm{Cs}_{2} \mathrm{CuCl}_{4}$ observed by neutron scattering, Phys. Rev. B 68, 134424 (2003).

[61] K. Foyevtsova, I. Opahle, Y.-Z. Zhang, H. O. Jeschke, and R. Valentí, Determination of effective microscopic models for the frustrated antiferromagnets $\mathrm{Cs}_{2} \mathrm{CuCl}_{4}$ and $\mathrm{Cs}_{2} \mathrm{CuBr}_{4}$ by density functional methods, Phys. Rev. B 83, 125126 (2011).

[62] S. A. Zvyagin, D. Kamenskyi, M. Ozerov, J. Wosnitza, M. Ikeda, T. Fujita, M. Hagiwara, A. I. Smirnov, T. A. Soldatov,
A. Y. Shapiro, J. Krzystek, R. Hu, H. Ryu, C. Petrovic, and M. E. Zhitomirsky, Direct Determination of Exchange Parameters in $\mathrm{Cs}_{2} \mathrm{CuBr}_{4}$ and $\mathrm{Cs}_{2} \mathrm{CuCl}_{4}$ : High-Field ElectronSpin-Resonance Studies, Phys. Rev. Lett. 112, 077206 (2014).

[63] R. Chen, H. Ju, H.-C. Jiang, O. A. Starykh, and L. Balents, Ground states of spin-1/2 triangular antiferromagnets in a magnetic field, Phys. Rev. B 87, 165123 (2013).

[64] M. Lee, J. Hwang, E. S. Choi, J. Ma, C. R. Dela Cruz, M. Zhu, X. Ke, Z. L. Dun, and H. D. Zhou, Series of phase transitions and multiferroicity in the quasi-two-dimensional spin-1/2 triangular-lattice antiferromagnet $\mathrm{Ba}_{3} \mathrm{CoNb}_{2} \mathrm{O}_{9}$, Phys. Rev. B 89, 104420 (2014).

[65] K. Yokota, N. Kurita, and H. Tanaka, Magnetic phase diagram of the $S=1 / 2$ triangular-lattice Heisenberg antiferromagnet $\mathrm{Ba}_{3} \mathrm{CoNb}_{2} \mathrm{O}_{9}$, Phys. Rev. B 90, 014403 (2014).

[66] Y. Shirata, H. Tanaka, A. Matsuo, and K. Kindo, Experimental Realization of a Spin-1/2 Triangular-Lattice, Phys. Rev. Lett. 108, 057205 (2012).

[67] J. Ma, Y. Kamiya, T. Hong, H. B. Cao, G. Ehlers, W. Tian, C. D. Batista, Z. L. Dun, H. D. Zhou, and M. Matsuda, Static and Dynamical Properties of the Spin-1/2 Equilateral TriangularLattice Antiferromagnet $\mathrm{Ba}_{3} \mathrm{CoSb}_{2} \mathrm{O}_{9}$, Phys. Rev. Lett. 116, 087201 (2016).

[68] S. Ito, N. Kurita, H. Tanaka, S. Ohira-Kawamura, K. Nakajima, S. Itoh, K. Kuwahara, and K. Kakurai, Structure of the magnetic excitations in the spin-1/2 triangular-lattice Heisenberg antiferromagnet $\mathrm{Ba}_{3} \mathrm{CoSb}_{2} \mathrm{O}_{9}$, Nat. Commun. 8, 235 (2017).

[69] Y. Cui, J. Dai, P. Zhou, P. S. Wang, T. R. Li, W. H. Song, J. C. Wang, L. Ma, Z. Zhang, S. Y. Li, G. M. Luke, B. Normand, T. Xiang, and W. Yu, Mermin-Wagner physics, $(H, T)$ phase diagram, and candidate quantum spin-liquid phase in the spin$1 / 2$ triangular-lattice antiferromagnet $\mathrm{Ba}_{8} \mathrm{CoNb}_{6} \mathrm{O}_{24}$, Phys. Rev. Mater. 2, 044403 (2018).

[70] Z. Zhu, P. A. Maksimov, S. R. White, and A. L. Chernyshev, Topography of Spin Liquids on a Triangular Lattice, Phys. Rev. Lett. 120, 207203 (2018).

[71] R. Kaneko, S. Morita, and M. Imada, Gapless spin-liquid phase in an extended spin 1/2 triangular Heisenberg model, J. Phys. Soc. Jpn. 83, 093707 (2014).

[72] Z. Zhu and S. R. White, Spin-liquid phase of the $S=1 / 2 \mathrm{~J} 1-\mathrm{J} 2$ Heisenberg model on the triangular lattice, Phys. Rev. B 92, 041105(R) (2015).

[73] Y. Iqbal, W.-J. Hu, R. Thomale, D. Poilblanc, and F. Becca, Spin liquid nature in the Heisenberg $J_{1}-J_{2}$ triangular antiferromagnet, Phys. Rev. B 93, 144411 (2016).

[74] W.-J. Hu, S.-S. Gong, W. Zhu, and D. N. Sheng, Competing spin-liquid states in the spin-1/2 Heisenberg model on the triangular lattice, Phys. Rev. B 92, 140403(R) (2015).

[75] S. Hu, W. Zhu, S. Eggert, and Y.-C. He, Dirac Spin Liquid on the Spin-1/2 Triangular Heisenberg Antiferromagnet, Phys. Rev. Lett. 123, 207203 (2019).

[76] E. A. Ghioldi, M. G. Gonzalez, S.-S. Zhang, Y. Kamiya, L. O. Manuel, A. E. Trumper, and C. D. Batista, Dynamical structure factor of the triangular antiferromagnet: Schwinger boson theory beyond mean field, Phys. Rev. B 98, 184403 (2018).

[77] F. Ferrari and F. Becca, Dynamical Structure Factor of the $J_{1}-J_{2}$ Heisenberg Model on the Triangular Lattice: Magnons, Spinons, and Gauge Fields, Phys. Rev. X 9, 031026 (2019).

[78] L. Chen, D.-W. Qu, H. Li, B.-B. Chen, S.-S. Gong, J. von Delft, A. Weichselbaum, and W. Li, Two-temperature scales in the 
triangular-lattice Heisenberg antiferromagnet, Phys. Rev. B 99, 140404(R) (2019).

[79] P. Czarnik, J. Dziarmaga, and P. Corboz, Evolution of an infinite projected entangled pair state: an efficient algorithm, Phys. Rev. B 99, 035115 (2019).
[80] D. Yamamoto, G. Marmorini, M. Tabata, K. Sakakura, and I. Danshita, Magnetism driven by the interplay of fluctuations and frustration in the easy-axis triangular $\mathrm{XXZ}$ model with transverse fields, Phys. Rev. B 100, 140410(R) (2019). 